\title{
Responsiveness to high intensity interval training depends on insulin sensitivity and protein content and composition of small extracellular vesicles
}

\section{Maria Apostolopoulou}

German Diabetes Center Duesseldorf, Germany

\section{Lucia Mastrototaro}

German Diabetes Center

\section{Sonja Hartwig}

German Diabetes Center Duesseldorf, Germany

\section{Dominik Pesta}

German Diabetes Center, Duesseldorf https://orcid.org/0000-0002-5089-3586

\section{Klaus Strassburger}

German Diabetes Center - Leibniz Institute for Diabetes Research at Heinrich Heine University Düsseldorf

\section{Elisabetta de Filippo}

German Diabetes Center Duesseldorf, Germany

\section{Tomas Jelenik}

German Diabetes Center Duesseldorf, Germany

\section{Yanislava Karusheva}

German Diabetes Center Duesseldorf, Germany

\section{Sofiya Gancheva}

German Diabetes Center, Duesseldorf

\section{Daniel Markgraf}

German Diabetes Center - Leibniz Institute for Diabetes Research at Heinrich Heine University Düsseldorf Christian Herder

German Diabetes Center https://orcid.org/0000-0002-2050-093X

\section{Sreekumar Nair}

Mayo Clinic

\section{Stefan Lehr}

German Diabetes Center - Leibniz Institute for Diabetes Research at Heinrich Heine University Düsseldorf

\section{Karsten Müssig}

German Diabetes Center - Leibniz Institute for Diabetes Research at Heinrich Heine University Düsseldorf

\section{Hadi Al-Hasani}

Clinical Research Centre, Department of Internal Medicine I, University Hospital Aachen, Aachen 
German Diabetes Center - Leibniz Institute for Diabetes Research at Heinrich Heine University Düsseldorf Michael Roden ( $\nabla$ michael.roden@ddz.de)

German Diabetes Center - Leibniz Institute for Diabetes Research at Heinrich Heine University Düsseldorf https://orcid.org/0000-0001-8200-6382

\section{Article}

Keywords: high intensity interval training, metabolism, insulin sensitivity, type 2 diabetes

Posted Date: December 2nd, 2020

DOI: https://doi.org/10.21203/rs.3.rs-105313/v1

License: (c) (i) This work is licensed under a Creative Commons Attribution 4.0 International License. Read Full License 


\section{Abstract}

High intensity interval training (HIIT) improves cardiorespiratory fitness (VO2max), but its impact on metabolism remains unclear. We hypothesized that 12-week HIIT improves insulin sensitivity in people with or without type 2 diabetes (T2D, NDM). However, despite identically improved VO2max, mainly insulin-resistant persons (T2D, IR NDM) responded with improved insulin sensitivity and circulating small extracellular vesicles (SEV), along with reduced myocellular protein kinase $\mathrm{C} \varepsilon$ activity (T2D) or inflammation (IR NDM). These changes related to the SEV proteome, characterized by downregulated phospholipase $C$ pathway (T2D) and upregulated antioxidant capacity (IR NDM). Thus, SEV cargo likely contributes to modulating exercise responsiveness in humans.

\section{Introduction}

Regular exercise training not only reduces cardiovascular risk, but also helps to prevent and treat type 2 diabetes $(T 2 D)^{1,2}$. However, up to $20 \%$ of all T2D participants in exercise interventions fail to respond to physical training with improved glucose metabolism ${ }^{3}$. Even first-degree relatives of persons with T2D do not necessarily increase their insulin sensitivity despite increased muscle ATP synthase flux after 6month exercise training ${ }^{4}$. Among other factors, exercise volume and intensity predict exercise responsiveness ${ }^{5}$. High intensity interval training (HIIT) represents a time-saving highly efficient alternative to moderate training modalities and may exert superior beneficial effects ${ }^{6}$, such as improved insulin sensitivity and mitochondrial function in elderly people ${ }^{7}$. However, 9-days HIIT failed to improve insulin sensitivity in insulin-resistant offspring of T2D despite increased mitochondrial function ${ }^{8}$. It remains to be determined whether longer term HIIT ameliorates mitochondrial changes and insulin sensitivity in insulin-resistant people with or without T2D.

Metabolic effects of exercise result from inter-tissue communication via metabolites, hormones, myokines/exerkines, microRNA or medium-sized extracellular vesicles ${ }^{9-12}$. Recent studies suggest that one exercise bout also leads to the release of small $(30-200 \mathrm{~nm}$ ) extracellular vesicles (SEV) by skeletal muscle in healthy volunteers ${ }^{13,14}$. However, the role of SEV in insulin-resistant humans and their impact on the metabolic response to a supervised exercise program per se, independent of diet or body weight changes, is not known. SEV can shuttle their functional cargo to target tissue to activate cellular signaling ${ }^{15}$, but it is also unclear whether and if so, which SEV cargo relates to changes in insulin sensitivity and underlying cellular pathways ${ }^{16}$.

Thus, this study examined the effect of a supervised 12-week HIIT on metabolic features and SEV release in sedentary insulin-sensitive (IS NDM) and insulin-resistant (IR NDM) glucose tolerant as well as T2D individuals. We originally hypothesized that HIIT would improve insulin sensitivity independently of glucose tolerance. Surprisingly, the responders to HIIT, as defined by improved peripheral insulin sensitivity (M-value), were mainly present among the insulin-resistant groups. This study therefore investigated next whether quantity and proteome of SEV help to differentiate between responders (T2D-R 
and IR-R from the T2D and IR NDM groups) and non-responders (IS-NR from the IS NDM group). SEV were isolated by size exclusion chromatography (SEC), allowing for subsequent downstream analysis ${ }^{17}$, and characterized by nanoparticle tracking analysis and mass spectrometry for protein identification and quantification. Finally, expression of selected SEV candidates was validated in skeletal muscle biopsies. This study revealed that HIIT indeed differently affects SEV concentration and their proteome depending on baseline insulin sensitivity, indicating that SEV cargo can modulate the metabolic responsiveness to exercise training.

\section{Results}

\section{Participants' characteristics at baseline}

Out of 49 persons, 20 T2D and 23 individuals without diabetes (NDM), completed the study (Fig. S1). Defined by a cutoff-value of $4.9 \mathrm{mg}^{*} \mathrm{~kg}^{-1} * \mathrm{~min}^{-1}$ for the M-value ${ }^{18}$, which reflects insulin-stimulated skeletal muscle glucose uptake ${ }^{16}, 11$ healthy persons were insulin-resistant (IR NDM) and 12 insulin-sensitive (IS NDM). Cardiorespiratory fitness $\left(\mathrm{VO}_{2} \mathrm{max}\right)$ was comparable between the three groups (Fig. 1a). M-value was lower in T2D and IR NDM than IS NDM ( $<<0.001$, Fig. 1b). Insulin-mediated suppression of endogenous glucose production (iEGP), reflecting hepatic insulin sensitivity was lower ( $p<0.001, \mathrm{Fig} .1 \mathrm{c}$ ), whereas liver fat content was higher in T2D than in both NDM groups $(p<0.001$, Fig. $1 d)$. As expected, T2D also had higher HbA1C and lower HDL-cholesterol than both NDM groups. Compared to IS NDM, T2D had higher body mass index (BMI), partly due to due to higher visceral fat mass (Table 1), whereas the higher BMI of IR NDM resulted from both higher subcutaneous and visceral fat mass.

\section{HIIT uniformly improves whole body oxidative and skeletal muscle mitochondrial capacity}

After 12-week of HIIT, all participants uniformly increased their $\mathrm{VO}_{2} \max$ (Fig. 1a) despite unchanged body weight and whole-body fat content (Table 1). Maximal uncoupled respiration, as assessed ex vivo from permeabilized skeletal muscle fibers tended to be lower in T2D than in IS NDM at baseline $(p=0.06)$, but rose by $28-45 \%$ in all groups after HIIT (Fig. 1e). HIIT neither affected leak control ratio (LCR), reflecting uncoupling at constant electron transport capacity, nor mitochondrial efficiency, assessed from respiratory control ratio (RCR), or reactive oxygen species (ROS) emission in any group (Fig. 1f,g). Muscle citrate synthase activity (CSA), as a surrogate marker of mitochondrial content ${ }^{19}$, at least doubled after HIIT in all groups without any difference between groups (Fig. 1h).

\section{Mainly insulin-resistant persons (T2D, IR NDM) exhibit increased peripheral insulin sensitivity upon HIIT}

Despite consistent increase in whole-body and skeletal muscle oxidative capacity, the response of Mvalue to HIIT substantially varied, separating groups of responders and non-responders. Logistic regression analysis showed an approximately doubled probability of the responder status among T2D $(16 / 20 ; 80 \%)$ and IR NDM $(9 / 11 ; 82 \%)$ as compared to IS NDM $(5 / 12 ; 42 \%)(\beta=1.94, p=0.02$ and $\beta=-2.2$, $p=0.03$, respectively). Likewise, only the insulin-resistant groups, T2D and IR NDM, improved their mean 
peripheral (Fig. 1b) and hepatic insulin sensitivity (Fig. 1C) as well as liver fat content (Fig. 1d). Only in T2D, HIIT also decreased visceral fat content, serum free fatty acids (FFA), triglycerides (TG) and showed a trend towards decreased glutamic-pyruvic transaminase (SGPT, $p=0.06)$ (Table 1).

\section{HIIT differently affects myocellular pathways of insulin sensitivity in the insulin-resistant responders (T2D-R, IR-R)}

To further examine the heterogeneous responses of the peripheral insulin sensitivity (M-value) within the groups, we employed skeletal muscle biopsies from subgroups of insulin-resistant responders, (T2D-R, $\mathrm{N}=16 ; \mathrm{IR}-\mathrm{R}, \mathrm{N}=9$ ) and insulin-sensitive non-responders (IS-NR, N=7) (Fig. 2a). We primarily addressed key mechanisms known to underlie human myocellular insulin resistance, such as abnormal mitochondrial function and inhibitory lipid signaling via activation of the diacylglycerol/novel protein kinase $\mathrm{C}$ isoform (DAG/nPKC) pathway and/or inhibitory inflammatory pathways ${ }^{16,20}$.

Similar to the whole groups, HIIT increased mitochondrial function (Fig. 2b), but did not affect ROS in all subgroups, although ROS was lower in IR-R than in T2D-R (Fig. 2C).

Activation of the $\mathrm{nPKC} \varepsilon$ and $\theta$ isoforms was assessed from translocation of the respective proteins to the myocellular membrane (Fig. S2a). PKC $\varepsilon$ activity negatively associated with M-value (log transformed; $\beta=-0.55 ; p=0.002$ ) at baseline, but not after HIIT. At baseline, PKC $\varepsilon$ activity was doubled in T2D-R compared to IR-R and IS-NR (Fig. 2d). After HIIT, T2D-R exhibited $50 \%$ and $40 \%$ decreases in PKC $\varepsilon$ and $\theta$ activities, respectively (Fig. 2d,e). Additional measurement of the nPKC activities in the whole groups of T2D, IR NDM and IS NDM, showed an identical pattern (Fig. S2b,c), indicating that the improvement in insulin resistance by HIIT is mediated by the NPKC pathway, at least in overt T2D.

Inflammatory pathways, specifically nuclear factor kappa-light-chain-enhancer of activated B cells (NF$K B)$, not only relate to insulin resistance and oxidative stress, but may be also downregulated by chronic exercising ${ }^{21,22}$. Thus, we also analyzed muscle NF-KB protein levels in all groups, which did not differ between responders and non-responders at baseline, but were reduced by $24 \%$ after HIIT exclusively in IR$R$ (Fig. 2f). This exercise effect was confirmed in the whole groups of T2D, IR NDM and IS NDM, although baseline NF-KB levels were 40\% higher in T2D (Fig. S2d). However, circulating pro- and anti-inflammatory cytokines (interleukin-1 $\beta,-6,-15,-1$ ra, tumor necrosis factor (TNF)a) were not significantly different between groups and after HIIT (data not shown). Collectively, these data suggest that the metabolic response to HIIT is at least partly mediated by reduction of $\mathrm{nPKC}$ activity in responders with diabetes (T2D-R) and by lower activation of NF-kB-dependent pathways in responders without diabetes (IR-R).

\section{HIIT induces an increase in circulating SEV in the insulin-resistant responders (T2D-R, IR-R)}

To clarify the role of SEV in exercise response, we measured the effect of HIIT on SEV release in representative subgroups of T2D-R ( $N=8), I R-R(N=8)$ and IS-NR $(N=6)$. These subgroups showed overall similar changes as the whole cohort, i. e. increased $\mathrm{VO}_{2}$ max in all, but increased peripheral and hepatic insulin sensitivity only in T2D-R and IR-R (Fig. S3a,b,c). Mitochondrial function increased in responders 
and was nominally higher compared to baseline in IS-NR (Fig. S3d), whereas ROS production remained unchanged in all three subgroups. Also, only T2D-R showed reduced PKC $\varepsilon$ activation (Fig. S3e), while only IR-R had lower NF-KB levels (Fig. S3f).

We measured the size and the number of circulating SEV in serum of responders and non-responders, collected at baseline and $72 \mathrm{~h}$ after the last bout of the 12-week HIIT. The purity of the SEV preparations was validated by nanoparticle tracking analysis (NTA) (Fig. 3a). The mean diameter of the isolated SEV was about $100 \mathrm{~nm}$ with a peak at about $80 \mathrm{~nm}$ and comparable between groups before and after HIIT (Fig. 3b). Interestingly, the estimated SEV concentration (number of circulating SEV per protein) was higher in IS-NR compared to T2D-R and numerically higher than in IR-R at baseline (Fig. 3). After HIIT, the SEV concentration rose only in the insulin-resistant groups, T2D-R and IR-R (Fig. 3d), with an increase of $52 \%$ (expressed as log2 fold change, FC) for both groups (Fig. S4a).

As HIIT increased the number of circulating SEV only in the insulin-resistant responders, we hypothesized that SEV and their cargo could contribute to the different cellular metabolic adaptations to this mode of chronic exercise training. We therefore characterized the proteome of the SEV isolated from T2D-R $(N=5)$, IR-R $(N=5)$ and IS-NR $(N=5)$ individuals at baseline and after HIIT and identified a total of 1707 proteins including the 24 exosomal-enriched proteins (Table S1). Furthermore, 1589 of the identified proteins (98\%) overlapped with the human exosome-associated proteins, previously identified in the Vesiclepedia database (13550 unique protein entries), whereas 39 were newly discovered as SEV-carried proteins (Fig. $4 a$, Table S2). Among the total SEV-associated proteins, we then selected the candidates with low variability (q value $<0.05$ ) and high degree of regulation (absolute log FC between groups or baseline vs HIIT >0.5) and we found that the proteomic profile differed between groups at baseline (Supplementary information) and after HIIT.

\section{HIIT affects the proteomic profile of SEV}

Quantitative proteomic analysis revealed that HIIT regulates the expression of 262 SEV proteins $(n=122$ in T2D-R, $n=130$ in IR-R, n=89 in IS-NR), of which 102 were downregulated and 160 upregulated (Fig. 4b, Table S3). Among the regulated SEV proteins, we identified proteins typically associated with exosomes $^{23}$, such as biogenesis markers (ALIX), signaling proteins (GTPase, Ras-related protein), proteins associated with membrane trafficking and fusion (Rab proteins, annexins), lipid rafts (flotilin), cytoskeleton components (moesin, tubulin) and cell adhesion molecules (integrins). Moreover, the cellular component (CC) enrichment analysis of regulated SEV proteins confirmed a significant enrichment of proteins associated with extracellular exosomes (Fig. 4c). Of note, 13 of the 262 SEV proteins, differentially expressed after HIIT, were shared between all groups (Fig. 4d, Table S3), including antithrombin III, kininogen I, histidine-rich glycoprotein and a1-antitrypsin, which relate to inflammatory and immune responses ${ }^{24}$. Moreover, 29 SEV proteins were up- or down-regulated after HIIT exclusively in responders, but not in IS-NR (Fig. 4d, Table S3). Among these proteins, fibrinogen a, b and g chains (FGA, FGB, FGG) were similarly upregulated in SEV isolated from T2D-R and IR-R after HIIT. These acute-phase proteins are not only associated with insulin resistance and acutely increased by insulin in T2D 25 , but 
have been also described as myokine candidates carried by EV and released from the exercising limb after recovery ${ }^{14}$, suggesting that exercise might activate insulin sensitizing pathways.

\section{Proteomic profiling of SEV suggests a new mode of myokine release for the metabolic adaptation to exercising}

Since 12-week HIIT triggered the release of SEV, we assumed that SEV might represent an alternative to the release of biologically active proteins by classical secretory pathway. Indeed, we found that only $34 \%$ $(89 / 262)$ of the proteins differentially expressed after HIIT had a predicted secretory signal peptide (SP) and only $12 \%$ (32/262) were predicted to follow a non-classical secretion pathway, based on the bioinformatics tools SignalP and SecretomeP (Fig. 4e, Table S3). These findings suggest that a large number of proteins can enter the circulation within SEV and that SEV can thereby contribute to inter-organ communication affecting cellular metabolism. In order to examine whether HIIT-induced increase in circulating SEV - at least partially - originated from skeletal muscle, we performed another quantitative proteomic analysis of SEV released from primary human skeletal muscle cells (hSkMC) using electrical pulse stimulation (EPS) to simulate the in vivo exercise intervention under in vitro conditions (Fig. 4f, Table S4). Interestingly, 44 SEV proteins regulated after HIIT were also present in SEV collected from the media of EPS-trained hSkMC, suggesting that these SEV proteins could represent novel myokines. Some of the SEV proteins upregulated, both after HIIT in vivo and after EPS in vitro, are indeed involved in signal transduction (GO:0007165; Ras-related protein Rap-2b and 1b), in oxidation-reduction processes (G0:0055114; aldehyde dehydrogenase family 16 member A1) and in cellular response to oxidative stress (G0:0034599; protein/nucleic acid deglycase DJ1,peroxiredoxin-2).

\section{HIIT enriches SEV with proteins related to insulin sensitivity in T2D-R and inflammation and oxidative metabolism in IR-R}

The 262 SEV proteins differentially regulated by HIIT were subsequently subjected to gene ontology (GO) analysis of biological processes (BP) and molecular function (MF) and to Ingenuity Pathway Analysis (IPA), as described in the supplementary information. In order to assess whether SEV protein cargo is responsible for the different metabolic adaptations induced by exercise in responders and nonresponders, we performed a functional analysis of the exercise-regulated SEV proteins in each group (T2D-R, IR-R and IS-NR) and found different enriched GO-terms and pathways (Table S5).

In T2D-R, functional analysis revealed a significant enrichment of proteins linked to glycolytic process and the inhibition of glycolysis (z-score -2), PLC (z-score -2), mitogen-activated protein kinase (ERK/MAPK) (z-score -2.236) and protein kinase A (PKA) signaling (z-score -2.249), since the proteins associated to these pathways were downregulated after HIIT (glycolysis: ENO1, PGK1, PKM, TPI1; ERK/MAPK: HSPB1, PRKACB, PRKAR1A, RAB1B, PRKCB; PLC: BTK, GNAQ, PRKCB, RAB1B; PKA: GNAQ, MYLK, PRKACB, PKACB, PRKAR1A, PRKCB, RAB1B) (Fig. 5a). In line with the pathways analysis, IPA identified MAPK1 and interleukin-15 (IL15) as upstream molecules inhibited after HIIT (z-score -2.000 for both). 
In IR-R, we found an overrepresentation of the non-canonical NF-KB and TNF-mediated pathways with upregulation of the 20S core proteasome complex (PSMA1, PSMA3, PSMA5, PSMA6, PSMA7, PSMB1, PSMB5, PSMB8) ${ }^{26,27}$ as well as the process "cellular response to oxidative stress" with an upregulation of proteins belonging to the cellular antioxidant system (CAT, CCS, G6PD, NME2, PRDX1, PRDX2, SOD2) (Fig. 5b). In line, IPA predicted the overrepresentation of pentose phosphate pathway, with an upregulation of glucose-6-phosphate dehydrogenase (G6PD), the rate-controlling enzyme of this pathway ${ }^{28}$, and the activation of the upstream molecule NFE2L2 (z-score 2.607) and the NRF2-mediated oxidative stress response ( $z$-score 2 ) since the downstream targets of NRF2 (CAT, PRDX1, PRKCA, RAB1B, SOD2, AKR7A2) were upregulated after HIIT. IPA revealed also IL 15 as activated upstream regulator (zscore 2.224), probably stimulated by the antioxidant system ${ }^{29}$. Finally, in the IR groups we found an enrichment of proteins associated to "response to calcium ion", extending the concept of the involvement of $\mathrm{Ca}^{2+}$ in $\mathrm{EV}$ release ${ }^{30}$ (Fig. $\left.5 \mathrm{a}, \mathrm{b}\right)$.

In IS-NR, we found a "lipid metabolism and transport" signature (Fig. 5c) and IPA revealed "autophagy of cells" as a cellular function associated only with SEV derived from IS-NR after 12-week HIIT (B-H p-value $<0.05$; z-score 1.432).

Taken together, these results indicate that HIIT (i) stimulates the insulin downstream pathway by inhibiting the MAPK, PLC and PKA signaling in the SEV from T2D-R and (ii) enhances the antioxidant system in the SEV released by IR-R, which eventually leads to improved peripheral insulin sensitivity, while (iii) it does not affect any pathway in SEV from IS-NR.

\section{SEV cargoed proteins released after HIIT may directly affect cellular pathways in skeletal muscle}

Finally, for investigating whether the specific SEV proteins also affect molecular pathways in skeletal muscle, we measured selected candidates related to insulin signaling for validation in muscle biopsies of T2D-R, IR-R and IS-NR individuals. Activating Thr172 phosphorylation of AMP activated protein kinase (AMPK) was higher in T2D-R than in IR-R at baseline and after HIIT (Fig. 6a). Inhibitory Ser307phosphorylation of insulin receptor substrate 1 (IRS1) was numerically lower in IR-R after HIIT $(p=0.13)$, whereas Ser1101-phosphorylation of IRS1 was not different between the groups (Fig. 6b,c). In addition, NRF2 and its downstream target NADPH quinone dehydrogenase 1 (NQO1) levels were lower at baseline in IR-R compared to T2D-R and tended to rise only in IR-R (Fig. 6d,e). After HIIT, also expression of both p38 mitogen-activated protein kinase (MAPK) and p44/p42 MAPK, as inflammation mediators target of $\mathrm{TNFa}^{31}$, were lower in IR-R and in IS-NR (Fig. 6f,g). Expression levels of microtubule-associated proteins 1A/1B light chain 3B (LC3) and ubiquitin-binding protein p62 (p62) was increased after HIIT only in IS-NR (Fig. 6h,i).

\section{Discussion}

This study found that (i) the majority of insulin-resistant, but not insulin-sensitive humans respond to HIIT with improved insulin sensitivity, (ii) the myocellular mechanism underlying the improvement differs 
between nondiabetic and diabetic responders (NF-KB vs. nPKC), (iii) only the responders also increase their circulating SEV and that (iv) the SEV proteome composition is differently affected between nondiabetic and diabetic responders (upregulated NRF2 vs. downregulated PLC, PKA and ERK pathways), probably mediating changes in muscle metabolism.

First, this study showed that HIIT similarly improves whole body and muscle maximal oxygen uptake and muscle mitochondrial mass, at least when assessed from CSA, in metabolically healthy as well as in insulin-resistant groups (IR NDM, T2D). This is in line with the exercise-induced increases in PGC1-a, mitochondrial density, oxidative capacity and mitochondrial complex proteins reported for glucosetolerant and T2D individuals ${ }^{32,33}$. Exhaustive exercising may not only increase oxidative capacity, but also cause oxidative stress with beneficial effects on mitochondrial adaptation and insulin sensitivity ${ }^{34}$. In the present study, HIIT did not induce oxidative stress in any group, but led to improved peripheral and hepatic insulin sensitivity in the insulin-resistant groups. Previous HIIT studies reported heterogeneous results in individuals with metabolic diseases albeit mostly using less accurate methods than the hyperinsulinemic-euglycemic clamp to assess insulin sensitivity ${ }^{35}$. In contrast, a study implying a twostep clamp protocol showed that HIIT improved peripheral insulin sensitivity but not EGP in young and elderly people with similar baseline insulin sensitivity ${ }^{7}$. Of note, one 8-week HIIT study found improved HOMA-IR in insulin-resistant humans ${ }^{36}$ and a 6-week intensive exercise intervention led to greater insulinstimulated muscle glucose transport/phosphorylation in insulin-resistant offspring of parents with T2D than in glucose tolerant humans ${ }^{37}$. These findings already indicate an ability of insulin-resistant individuals to (over)compensate for insulin resistance by adequate exercise training.

Of note, it has been estimated that $20 \%$ of T2D are non-responders to exercise ${ }^{3}$ and $31 \%$ do not improve their metabolic control and insulin sensitivity after hypocaloric dietary intervention ${ }^{38}$. Genetic predisposition could play an important role regarding response to lifestyle interventions, as reported in several studies ${ }^{39,40}$. For example, non-response among glucose-tolerant relatives of parents with T2D has been attributed to a polymorphism in the NDUFB6 gene, which encodes a subunit of mitochondrial complex ${ }^{41}$, supporting the concept of a tight association between mitochondrial function and changes in insulin sensitivity in T2D patients ${ }^{33}$. Of note, the present study found that mainly insulin-resistant individuals responded to HIIT with improved insulin resistance independently of muscle mitochondrial function. In this context, another study reported that non-responders to exercise training also had higher baseline insulin sensitivity along with different DNA methylation status and RNA expression patterns ${ }^{42}$.

Interestingly, the present study showed that the improvement of insulin resistance upon HIIT associated with different pathways in T2D and IR NDM. More specifically, in T2D -but not in IR NDM- we found a reduction of $\mathrm{nPKC} \varepsilon$ and $\theta$ activities. This indicates that DAG/nPKC pathway, which is well known to mediate lipid-induced insulin resistance ${ }^{16,43}$, is downregulated by HIIT at least in overt diabetes. In line, HIIT also led to reductions in liver fat content paralleled with improved hepatic insulin sensitivity in both T2D and IR NDM and these changes were in contrast to divergent effects of HIIT in non-alcoholic fatty liver disease (NAFLD) ${ }^{44}$. 
The T2D group also showed activation of the NF-KB pathway, which is another feature of long-standing obesity and T2D ${ }^{31}$. HIIT reduced NF-KB protein expression in IR NDM, but not in T2D, indicating the operation of different mechanisms of metabolic exercise response in individuals with or without diabetes. One might suggest that improvement of the more severe insulin resistance requires reduction of the lipotoxic pathways in skeletal muscle. Of note, the reduction in myocellular inflammatory pathways in IR NDM was not accompanied by any changes in circulating inflammatory markers, suggesting that lowgrade (subclinical) inflammation may not be the primary inter-organ crosstalk mechanism explaining HIIT-induced metabolic changes.

To elucidate other mechanisms of inter-organ crosstalk mediating the metabolic effects of $\mathrm{HIIT}^{12}$, we isolated circulating SEV and found that their number was already lower in insulin-resistant responders than in BMI-matched insulin-sensitive non-responders before exercise training. This is in contrast to a recent study reporting higher number of $E V$ in T2D than lean healthy humans ${ }^{45}$, which may be explained by the differences in BMI between the groups, since circulating EV are significantly increased in obesity ${ }^{46}$. In addition, the previous study reported an increase of total (large and small vesicles) and large EV, but not specifically of SEV due to a different method of vesicle isolation.

Previous studies have only reported on the acute release of EV upon a single exercise bout in healthy mice and humans ${ }^{12-14}$. This study shows that 12-week HIIT induces secretion of SEV in insulin-resistant humans responding with improved insulin resistance (IR-R and T2D-R). Also, several SEV proteins regulated after HIIT were found also in exosomes released from hSkMC after EPS in vitro, supporting the concept that skeletal muscle is likely the major contributor to exercise-induced SEV release. The large skeletal muscle mass, its central involvement in exercise responses and high secretory activity in terms of myokines may serve to support this contention ${ }^{47}$.

Analysis of SEV proteins differentially expressed after HIIT in T2D-R revealed inactivation of PLC, PKA and ERK/MAPK signaling, which can be translated into a functional IRS-PI3K/Akt-GLUT4 signaling pathway and improved insulin sensitivity ${ }^{16,48}$. Indeed, inactivation of PLC and PKA in SEV might serve as link to the reduced $\mathrm{nPKC}$ activity and the upregulated AMPKa activity observed in skeletal muscle of T2DR. Although we did not verify pathways in other target tissues, it is conceivable that SEV mediate metabolic improvements also in other organs, such as liver, and contribute with its cargo to improved hepatic insulin sensitivity observed in T2D-R via inhibition of PKC $\varepsilon$ and activation of AMPKa, as previously described in PKC $\varepsilon$ knock-out mice ${ }^{49}$ and primary hepatocytes treated with metformin ${ }^{50}$.

In IR-R, the SEV proteome after HIIT displayed an upregulation of the 20S core proteasome complex of the non-canonical NF-KB and TNF-mediated pathways, which reduce inflammation via degradation of NF$K B$ and transcriptional termination of target genes ${ }^{26,27}$, and activation of the NRF2-mediated oxidative stress response, which potentiate antioxidative response in target tissues. Of note, skeletal muscle of IR-R showed reduced NF-KB, p38 and p44/p42 MAPK proteins and upregulation of NRF2 and NQ01 proteins, which decrease the inhibitory Ser307-phosphorylation of IRS1 and protect against insulin resistance ${ }^{16,51}$. 
SEV proteins might reduce activation of NF-KB also in liver and thereby reduce hepatic inflammation, insulin resistance and fat content ${ }^{52}$, as observed in IR-R after HIIT.

Finally, SEV proteome isolated from IS-NR did not reveal any significant inhibition or activation of pathways in line with the non-responder status, i. e. the absence of changes in insulin sensitivity. However, expression of the autophagy markers, LC3 and p62, was markedly increased after 12-week HIIT in their skeletal muscle, which may reflect enhanced endurance performance ${ }^{53}$.

Taken together, this study shows that (i) even highly efficient HIIT exercise training does not generally improve peripheral insulin sensitivity, but allows to identify responders, who are more likely insulinresistant humans at baseline, (ii) exercise response of insulin sensitivity relates to different mechanisms among individuals with or without T2D, i. e. reduction of myocellular lipotoxic signaling via DAG/nPKC or downregulation of NF-KB pathways and that (iii) different amount and proteome of circulating SEV likely contribute to the variation in metabolic exercise response. These findings might help to better understand mechanisms of lifestyle intervention and to identify novel targets for the tailored prevention and treatment of insulin resistance and T2D in the concept of precision medicine.

\section{Online Methods}

\section{Study participants and study design}

This study included 20 male patients with type 2 diabetes (T2D) as well as 12 age-matched insulinsensitive (IS) and 11 insulin-resistant (IR) glucose tolerant male participants (NDM). All participants underwent a screening procedure with detailed physical examination, interview, blood sampling and spiroergometry. Exclusion criteria were performance of more than 60 minutes of endurance training per week, acute or chronic cardiovascular, renal or liver diseases, use of insulin-sensitizing medication or beta-blockers, alcohol intake of more than $30 \mathrm{~g}$ per day and smoking. Volunteers of the control group needed to have neither any family history of T2D nor dysglycemia during a standardized 75-g oral glucose tolerance test. All participants gave written informed consent prior to inclusion in the study, which was approved by the institutional review board of Heinrich-Heine University Düsseldorf (NCT02039934) and performed according to the World's Medical Association Declaration of Helsinki.

\section{Exercise training protocol and diet control}

All participants took part in a progressive 12-week supervised cycle ergometer training protocol of a total duration of 35 minutes per session consisting of four 4-minute high-intensity intervals (HIIT), during which the participant trained at an intensity corresponding to $90 \%$ of their maximal heart rate as determined during a baseline spiroergometry. These intervals were interspersed by three 3-minute intervals, during which the participant trained at $70 \%$ of their maximal heart rate. Exercise training sessions were repeated three times weekly on non-consecutive days. Over the 12-week training period, workload was progressively adjusted to maintain the target heart rate. All participants were instructed to maintain their body weight (change up to $5 \%$ of initial body weight was tolerated) throughout the training 
period. In turn, body weight was checked every 4 weeks and participants presenting with a $3 \%$ change of their initial body weight received adequate dietary counselling by our expert clinical nutritionists.

\section{Spiroergometry}

Each participant performed an incremental exhaustive exercise test on an electronically braked cycle ergometer (Ergoline ergometrix 900, Bitz, Germany) at 60 revolutions $/ \mathrm{min}^{54}$. Respiratory gas exchange measurements were determined by open-air spirometry (Masterscreen CPX; Jäger/Viasys, Hoechberg, Germany) ${ }^{55}$. During exercising, work rate was continually increased by $15 \mathrm{~W} / \mathrm{min}$ increments, and the incremental part of the test lasted 8-12 minutes. Blood pressure, heart rate and a 12-lead electrocardiogram (ECG) were recorded every 2 minutes during the test. Capillary blood was drawn from the ear every 2 minutes and then every minute after the anaerobic threshold for the measurement of lactate.

\section{Hyperinsulinemic-euglycemic clamp tests}

Two-step hyperinsulinemic-euglycemic clamp tests were performed for assessment of peripheral and hepatic insulin sensitivity before intervention and $72 \mathrm{~h}$ after the last training session. Patients with T2D did not take their oral glucose lowering medication for three days before the clamp to avoid any interference from metabolic drug effects. The clamp test were performed as previously described in detail ${ }^{56}$. On the day of test, participants arrived at the clinical research center at 07:00 a.m., where they received two venous catheters in the antecubital veins of both arms for blood sampling and infusions of glucose and insulin. A primed-continuous infusion of $98 \%$ enriched D-[6,6- $\left.{ }^{2} \mathrm{H}_{2}\right]$ glucose was initiated at minute 0 and continued until the end of the clamp test to measure endogenous glucose production (EGP). After $120 \mathrm{~min}$, a low-dose primed-continuous infusion $\left(20 \mathrm{mU} /\left[\mathrm{m}^{2}\right.\right.$ body surface area*min] $)$ of insulin (Actrapid; Novo Nordisk, Copenhagen, Denmark;) was administered, followed by a higher dose primed-continuous infusion ( $40 \mathrm{mU} /\left[\mathrm{m}^{2}\right.$ body surface area*min]) from 120 to $240 \mathrm{~min}^{56}$. A continuous somatostatin infusion was administered throughout the clamp in order to inhibit endogenous insulin secretion. Blood glucose measurements were performed every 5 minutes and a $20 \%$ dextrose infusion labeled with $\mathrm{D}-\left[6,6{ }^{2} \mathrm{H}_{2}\right]$ glucose ( $2 \%$ enriched) was adjusted to maintain normoglycemia $(5 \mathrm{mmol} / \mathrm{l})$. Peripheral insulin sensitivity was measured from mean whole-body glucose infusion rates (M-value) during the last 30 minutes of the clamp with glucose space correction.

\section{Laboratory measurements}

All parameters were obtained in the morning of test day and after $10 \mathrm{~h}$ of fasting ${ }^{54}$. Blood glucose, serum triglycerides, high- and low- density lipoproteins and glutamic pyruvic transaminase (SGPT) were measured on a Cobas c311 (Roche Diagnostics, Mannheim, Germany). Serum concentrations of insulin and C-peptide were measured with RIA (Millipore, St Charles, MO, USA) and free fatty acids (FFA) microfluorimetrically. 


\section{Proton magnetic resonance spectroscopy ('H-MRS)}

Liver fat content was quantified by volume selective proton magnetic resonance spectroscopy ( $\left.{ }^{1} \mathrm{H}-\mathrm{MRS}\right)$ using a stimulation echo acquisition mode in a whole-body 3-Tesla magnetic resonance scanner, as described ${ }^{57}$. Whole-body, subcutaneous and visceral fat mass were quantified by application of a T1weighted axial fast spin echo ${ }^{54}$.

\section{High resolution respirometry}

Mitochondrial respiration was assessed in permeabilized muscle fibers from skeletal muscle biopsies ${ }^{43}$. Leak respiration (LEAK) after addition of glutamate and malate, state 3 respiration at saturating ADP concentrations (OXPHOS), LEAK state induced by inhibition of ATP synthase by oligomycin (LEAKomy) and electron transport (ET) capacity to obtain maximum oxygen flux at optimum concentration of the uncoupler carbonyl cyanide-4-(trifluoromethoxy)phenylhydrazone (FCCP) were assessed in a 2-chamber Oxygraph-2k (OROBOROS Instruments, Innsbruck, Austria). Respiratory control ratio (RCR) was calculated as the ratio of OXPHOS/LEAKomy, whereas leak control ratio (LCR) was calculated from the ratio of LEAK/ET capacity.

\section{Skeletal muscle biopsy}

At baseline as well as after 12-week HIIT, a skeletal muscle biopsy was performed on the day of clamp procedure, directly before initiation of insulin infusion. The region above the muscle vastus lateralis was anesthetized using local anesthetics (Lidocain $2 \%$ ) and $100-500 \mathrm{mg}$ of skeletal muscle tissue was obtained using a Bergstrom needle ${ }^{43}$. An aliquot of $50 \mathrm{mg}$ of tissue was transferred into ice-cold biopsy preservation solution (BIOPS) for high resolution respirometry and the rest was stored in liquid nitrogen and frozen at $-80^{\circ}$ until further analysis.

\section{Citrate synthase assay}

Citrate synthase activity (CSA) was assayed spectrophotometrically by a commercial kit (Citrate Synthase Assay Kit; Sigma-Aldrich, St. Louis, MO, USA), according to the method of Morgunov and Srere ${ }^{58}$. CSA for each sample was normalized to protein concentration, which was determined in the supernatant using the BCA (bicinchoninic acid) Assay Kit (Thermo Scientific, Dreieich, Germany).

\section{Western blot}

Expression levels of proteins of interest were assessed with western blot ${ }^{59}$. Proteins were extracted from approximately $30 \mathrm{mg}$ of frozen skeletal muscle and homogenized in $300 \mu \mathrm{l}$ of lysis buffer $(25 \mathrm{mM}$ trisaminomethane-hydrochloride (Tris- $\mathrm{HCl}$ ), $1 \mathrm{mM}$ ethylenediaminetetraacetic acid (EDTA), $150 \mathrm{mM} \mathrm{NaCl}$, $0.20 \%$ NP-40) with protease (cOmplete Tablets, EASYpack, Roche Diagnostics) and phosphatase (PhosSTOP, EASYpack, Roche Diagnostics) inhibitors for extraction of total soluble proteins. Samples were shaken 3 times $1 \mathrm{~min}$ at $20 \mathrm{~Hz}$ in a Tissue Lyzer and centrifuged $\left(13000 \mathrm{rpm}, 15 \mathrm{~min}, 4^{\circ} \mathrm{C}\right)$ to pellet 
insolubilized material, nuclei, unbroken cellular membranes. Activities of PKC $\theta$ and PKC $\varepsilon$ were assessed from the ratios of the protein contents in membrane and cytosol fractions upon differential centrifugation. The tissue was homogenized in $300 \mu \mathrm{l}$ of lysis buffer A ( $25 \mathrm{mM}$ Tris-HCL, $1 \mathrm{mM}$ EDTA, 150 $\mathrm{mM} \mathrm{NaCl}, 0.20 \% \mathrm{NP}-40$ with protease and phosphatase inhibitors). The homogenate was centrifuged $\left(100000 \mathrm{~g}, 1 \mathrm{~h}\right.$ at $\left.4^{\circ} \mathrm{C}\right)$ and the supernatant containing the cytosolic fraction was transferred to a fresh tube and frozen at $-80^{\circ} \mathrm{C}$, while the pellet was dissolved in $110 \mu \mathrm{l}$ of buffer $\mathrm{B}(250 \mathrm{mM}$ Tris-HCL, $1 \mathrm{mM}$ EDTA, $0.25 \mathrm{mM}$ EGTA, 2\% Triton X-100) using a homogenizer. A second centrifugation step (100000 g, $1 \mathrm{~h}$ at $4{ }^{\circ} \mathrm{C}$ ) was performed and the supernatant (membrane fraction) was collected and stored at $-80^{\circ} \mathrm{C}$ as well.

The concentration of the extracted proteins was determined in the supernatant using the BCA Assay Kit. Aliquots of $30 \mu \mathrm{g}$ of total proteins, as well as cytosolic and membrane fractions, were diluted 4 times with the loading buffer $(0.35 \mathrm{M}$ Tris- $\mathrm{HCl}$ at $\mathrm{pH}$ of $6.8,10 \%$ sodium dodecyl sulfate (SDS), $30 \%$ glycerol, $0.6 \mathrm{M}$ dithiothreitol (DTT), $0.175 \mathrm{mM}$ Bromophenol Blue) and then loaded onto a SDS-polyacrylamide gradient gel (4-20\% Mini-PROTEAN® TGX ${ }^{\mathrm{TM}}$ Precast Protein Gels, Biorad, CA, USA). Following electrophoresis, a semi-dry blotting to a polyvinylidene difluoride (PVDF) membrane was performed at $8 \mathrm{~mA} / \mathrm{cm}^{2}$ for $1 \mathrm{~h}$. After blocking the membranes for $2 \mathrm{~h}$ at room temperature using the blocking solution ( $5 \%$ milk in Trisbuffered saline-Tween (TBS-T)), the membranes were incubated with the primary antibodies diluted 1:1000 in blocking solution in combination with the respective horseradish peroxidase (HRP)-conjugated secondary anti-rabbit antibody, diluted 1:2500, or anti-mouse diluted 1:1000. The membranes were finally coated with Immobilon Western Chemiluminescent HRP Substrate (Millipore) and the proteins were detected using a Bio-Rad ChemiDocTM MP Imaging System in combination with the software ImageLab 6.0.1 (Bio-Rad 199 Laboratories) for densitometric analysis. Primary antibodies were purchased from Cell Signaling Technology: LC3 (4108); p38 MAPK (9212); p44/p42 MAPK (9102); NF-KB (8242); AMPKa (2532); phospho-AMPKa(Thr172) (2535); NQ01 (3187); phospho-IRS1(Ser307) (2381); phosphoIRS1(Ser1101) (2385); GAPDH (glyceraldehyde 3-phosphate dehydrogenase) (2118) as housekeeping protein for the soluble and cytosolic fractions. p62 (610833), PKC $\theta$ (610090) and PKC $\varepsilon$ (610086) were obtained from BD Biosciences; IRS1 from Millipore (06-248), NRF2 from Santa Cruz Biotechnology (sc365949) and $\mathrm{Na}^{+} / \mathrm{K}^{+}$-ATPase, used as loading control for the membrane fraction, from Abcam (Ab76020). Data are expressed in arbitrary units and normalized to housekeeping protein.

\section{Cell culture of primary human skeletal muscle cells (hSkMC)}

Proliferating myoblasts of three healthy Caucasian donors (male, 16, 21 and 41 years of age [M16, M21 and M41]) were obtained to generate hSkMC (PromoCell, Heidelberg Germany) and cultured as described $^{60}$. For an individual experiment, myoblasts were seeded in six-well culture dishes and cultured to $90 \%$ of confluence in a-modified Eagle's medium (aMEM)/Ham's F-12 medium (Gibco, Berlin Germany) containing supplement for skeletal muscle cell growth medium (PromoCell). The cells were then differentiated in aMEM supplemented with $2 \%$ horse serum for 5 days, followed by overnight starvation in 
aMEM without serum. Differentiated cells were subjected to electrical pulse stimulation (EPS) cells ${ }^{60}$ and after $24 \mathrm{~h}$ of EPS the SEV were isolated from conditioned medium as reported ${ }^{61}$.

\section{Small extracellular vesicles (SEV) isolation from serum}

Serum samples were collected before the intervention and $72 \mathrm{~h}$ after the last training. Small extracellular vesicles were isolated from serum by size exclusion chromatography, using the IZON columns (qEV2/70 $\mathrm{nm}$ ) coupled to successive ultracentrifugation ${ }^{62}$. Briefly, the serum samples (starting material $750 \mu \mathrm{l}$ ) were first cleared at $1500 \mathrm{~g}$ for $10 \mathrm{~min}$ followed by centrifugation at $10000 \mathrm{~g}$ for $10 \mathrm{~min}$ to remove particulate matter. The serum supernatant was then loaded into an IZON column previously equilibrated with PBS. Once the serum entered the column, $50 \mathrm{ml}$ of PBS were loaded on top of the column. The first $13 \mathrm{ml}$ of flow-through were discarded and the elution fractions $14-21 \mathrm{ml}$ were collected. $300 \mu \mathrm{l}$ of PBSeluted EV were used for Nanoparticles Tracking Analysis, while the rest was centrifuged at $100000 \mathrm{~g}$ for $70 \mathrm{~min}$. The supernatant was discarded and the transparent pellet containing SEV was lysed for mass spectrometry sample processing.

\section{Nanoparticle tracking analysis (NTA)}

Count and size of SEV were assessed by NTA, which measures in real time the Brownian motion of vesicles resuspended in fluid. A Nanosight NS300 (Malvern Panalytical) with a $488 \mathrm{~nm}$ laser and the NTA 3.3 Software was used to detect the SEV by laser light scattering. Samples dissolved in PBS were diluted with $0.22 \mu \mathrm{m}$ filtered PBS to achieve 25-80 particles/frame. Data were collected from $3 \times 90 \mathrm{sec}$ videos recorded at a constant temperature of $25^{\circ} \mathrm{C}$ with viscosity $0.9 \mathrm{cP}$, camera level 16 and detection threshold 4.

\section{Mass spectrometry (MS)}

Extracellular vesicles pellets derived from human serum samples and skeletal muscle cells were lysed in denaturing SDS buffer ( $62.5 \mathrm{mM}$ Tris-HCl pH 6.8, 10\% glycerol, 2 mM EDTA, 2\% SDS and $100 \mathrm{mM}$ DTT) and loaded onto SDS-PAGE (10\% polyacrylamide, $0.5 \mathrm{~cm}$ separation distance) as previously described ${ }^{61}$. Subsequent to protein quantification of the Coomassie blue stained protein bands against a BSA standard, bands were excised and subjected to in-gel protein digestion. Therefore, gel slices were alternately washed twice with $25 \mathrm{mM}$ ammonium bicarbonate and $25 \mathrm{mM}$ ammonium bicarbonate and $50 \%(\mathrm{v} / \mathrm{v})$ acetonitrile (ACN). Protein reduction was performed in $65 \mathrm{mM}$ DTT for $15 \mathrm{~min}$, shaking at 350 $\mathrm{rpm}$ and $50^{\circ} \mathrm{C}$. Proteins were then alkylated in $216 \mathrm{mM}$ iodoacetamide for $15 \mathrm{~min}$ in the dark at room temperature. Followed by an additional washing step $(25 \mathrm{mM}$ ammonium bicarbonate, $25 \mathrm{mM}$ ammonium bicarbonate, $50 \%$ ACN (v/v)), gel slices were shrinked in 100\% (v/v) ACN. Digestion was performed with $100 \mathrm{ng}$ trypsin (Promega) in $25 \mathrm{mM}$ ammonium bicarbonate and $2 \%$ (v/v) ACN over night at $37^{\circ} \mathrm{C}$. Resulting peptides were eluted first with $1 \%(\mathrm{v} / \mathrm{v})$ trifluoroacetic acid (TFA) followed by elution with $0.1 \%$ TFA $/ 90 \%(\mathrm{v} / \mathrm{v}) \mathrm{ACN}$ and lyophiliation. For MS analysis, lyophilised peptides were reconstituted in $1 \%$ TFA ( $(\mathrm{v} / \mathrm{v})$ including iRT (indexed retention time) peptides (Biognosys) and separated by liquid chromatography (Ultimate 3000, ThermoFisher Scientific) using an EASYspray ion source equipped to an 
Orbitrap Fusion Lumos mass spectrometer (ThermoFisher Scientific). Peptides were trapped and desalted on an Acclaim PepMap C18-LC-column (ID: 75 um, 2 cm length; ThermoFisher Scientific) and

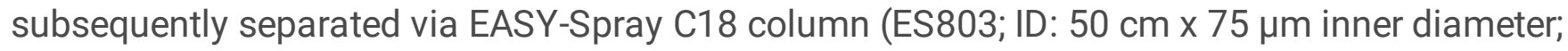
ThermoFisher Scientific) using a 100 min linear gradient from buffer A (0.1\% formic acid) to 4-34\% buffer $B(80 \%$ ACN, $0.1 \%$ formic acid) at a flow rate of $300 \mathrm{nl} / \mathrm{min}$ followed by a 20 min linear gradient increasing buffer B to $50 \%$ and a 1 min linear gradient increasing buffer B to $90 \%$. Column temperature was set to $40 \mathrm{C}^{\circ}$.

MS data for generating spectral libraries were acquired on an Orbitrap Fusion Lumos instrument (ThermoFisher Scientific) operated in data dependent mode. MS spectra were obtained at 120,000 resolution (3 s cycle time), $\mathrm{m} / \mathrm{z}$ range of 350-1600 and a target value of $4^{5}$ ions, with maximum injection time of $50 \mathrm{~ms}$. For fragmentation precursor selection filter were set to charge state between 2 and 7 , dynamic exclusion of $30 \mathrm{~s}$ and intensity threshold of $2.5^{4}$. Fragmentation of precursors was done with an isolation window (m/z) 1.2, HCD (Higher-energy collisional dissociation) energy of 32\%, Orbitrap resolution of 15,000 and an ion target value of $1.0^{5}$ with maximum injection time of $50 \mathrm{~ms}$.

MS data for label free quantification were acquired in a DIA (data independent acquisition) mode. Full scan MS spectra were obtained at 120,000 resolution, $\mathrm{m} / \mathrm{z}$ range of 400-1200, and an AGC (automatic gain control) target value of $5^{5}$ and maximum injection time of $50 \mathrm{~ms}$. Fragmentation was performed with HCD energy of 32\% in 34 windows cover the range from 400-1200 with a segment width of 24.5 $(\mathrm{m} / \mathrm{z})$, Orbitrap resolution of 30,000, AGC Target of $1.0^{6}$, scan range from $200-2000(\mathrm{~m} / \mathrm{z})$ and maximal injection time was $60 \mathrm{~ms}$.

\section{Analysis of mass spectrometry data}

DDA (data dependent acquisition) and DIA data were imported into Spectronaut Pulsar (Version 12, Biognosys) to generate a sample specific library using the default settings (modifications: carbamidomethyl (C) (fixed); oxidation (M), acetyl (protein N-term) (variable); enzyme: Trypsin/P; max. missed cleavages: 2) ${ }^{63}$. Pulsar search was done against a human FASTA file (UniProtKB database, reviewed SwissProt, Homo sapiens TaxID 9096 canonical and isoforms, downloaded 07-2018), results were filtered by an FDR of $1 \%$ on precursor and protein group level ( $q$ value $<0.01$ ).

For quantitative analysis the DIA data were analyzed in Spectronaut (Version 12, Biognosys) using the generated library (obtained from the corresponding DDA and DIA runs) with default settings. Candidate list was created using an average log2 ratio $\leq 0.58$ and $q$ value $\leq 0.05$.

\section{Bioinformatics analysis}

The proteins information was extracted from Swiss-Prot database (http://www.uniprot.org/). The prediction of a secretory signal peptide (SP) was done with the server SignalP 4.1 using as cutoff $0.450^{64,65}$ and the proteins without a SP were further analyzed with SecretomeP 2.0 to identify nonclassically secreted proteins (score $>0.6)^{64}$. The comparison between our study and the vesicle database 
Vesiclepedia was done using FunRich (v3.1.3; released on 2018) ${ }^{65}$. Gene ontology (GO) enrichment analysis of the SEV proteins regulated during exercise was performed for cellular component (GO-CC), molecular function (GO-MF) and biological process (GO-BP) versus annotations derived from Uniprot, with cutoff enriched $p$-value $<0.05$ (Benjamini-Hochberg, B-H, corrected FDR value).

In addition, the proteomic dataset, with UniProt identifiers, FC 12-week HIIT vs baseline and q value of each comparison, were uploaded into Ingenuity Pathway Analysis (IPA, Ingenuity ${ }^{\mathrm{Tm}}$, Qiagen, Hilden, Germany) for core analysis and overlaid with the Ingenuity pathways knowledgebase, in order to categorize the SEV-cargoed proteins in biological functions and pathways. IPA predicted also the upstream regulators for the proteins in the dataset, which are responsible for the pathways and networks most significant to the dataset.

\section{Statistical analysis}

Data are presented as mean and standard error of mean ( \pm SEM) or median (1st and 3rd quartile). Variables with a skewed distribution (M-value, triglycerides, liver fat content, SEV number, protein levels) were log-transformed before analysis. ANCOVA-like linear regression analyses of baseline to 12-week HIIT changes allowing for different residual variances between the investigated groups and t-tests were performed. Logistic regression analysis was used to estimate the probability of responder-status in each group and to investigate associations between PKC activity and M-value.

Analysis in IPA were performed using the B-H method of multiple testing correction, based on the Fisher's exact test $p$-value with a threshold value of 0.05 , and the $z$-score algorithm, which predicts the activation or inhibition of pathways and functions after HIIT according to the molecule expression changes in our dataset. We considered only functions and pathways with a z-score $>2$ or $<2$.

The B-H corrected p-value (threshold $<0.05$ ) was used also to determine significantly enriched GO-terms. Duplicate or highly similar GO-terms were removed and only those with the highest statistical power or with the highest number of genes in the background data set were selected. GraphPad Prism 8 was used to plot the graphs when comparing datasets (GraphPad Software, Inc).

\section{Declarations}

\section{Acknowledgments}

We would like to thank all volunteers, Agnieszka Sutkowski, Kai Tinnes and Myrko Esser for their excellent technical support, Martin Röhling for contributing to the clinical study and training supervision. Last, we would like to pay our gratitude and our respect to our colleague Dr. H. Sell who contributed to this study and passed away in September 2019. This study was supported in part by the Ministry of Culture and Science of the State of North Rhine-Westfalia (MKW NRW), the German Federal Ministry of Health (BMG) as well as by a grant of the Federal Ministry for Research (BMBF) to the German Center for Diabetes 
Research (DZD e. V.), the German Research Foundation (DFG, CRC 1116/2), German Diabetes Association (DDG) as well as the Schmutzler-Stiftung.

MR is the guarantor of this work and, as such, had full access to all the data in the study and takes responsibility for the integrity of the data and the accuracy of the data analysis. There are no conflicts of interest for this manuscript.

\section{References}

1. Chudyk, A. \& Petrella, R.J. Effects of exercise on cardiovascular risk factors in type 2 diabetes: a meta-analysis. Diabetes care 34, 1228-1237 (2011).

2. Colberg, S.R., et al. Physical Activity/Exercise and Diabetes: A Position Statement of the American Diabetes Association. Diabetes care 39, 2065-2079 (2016).

3. Stephens, N.A. \& Sparks, L.M. Resistance to the beneficial effects of exercise in type 2 diabetes: are some individuals programmed to fail? J Clin Endocrinol Metab 100, 43-52 (2015).

4. Kacerovsky-Bielesz, G., et al. A single nucleotide polymorphism associates with the response of muscle ATP synthesis to long-term exercise training in relatives of type 2 diabetic humans. Diabetes care 35, 350-357 (2012).

5. Sparks, L.M. Exercise training response heterogeneity: physiological and molecular insights. Diabetologia 60, 2329-2336 (2017).

6. Gibala, M.J., Little, J.P., Macdonald, M.J. \& Hawley, J.A. Physiological adaptations to low-volume, high-intensity interval training in health and disease. The Journal of physiology $590,1077-1084$ (2012).

7. Robinson, M.M., et al. Enhanced Protein Translation Underlies Improved Metabolic and Physical Adaptations to Different Exercise Training Modes in Young and Old Humans. Cell metabolism 25, 581-592 (2017).

8. Irving, B.A., Short, K.R., Nair, K.S. \& Stump, C.S. Nine days of intensive exercise training improves mitochondrial function but not insulin action in adult offspring of mothers with type 2 diabetes. $J$ Clin Endocrinol Metab 96, E1137-1141 (2011).

9. Herder, C., Carstensen, M. \& Ouwens, D.M. Anti-inflammatory cytokines and risk of type 2 diabetes. Diabetes Obes Metab 15 Suppl 3, 39-50 (2013).

10. Molnos, S., et al. Metabolite ratios as potential biomarkers for type 2 diabetes: a DIRECT study. Diabetologia 61, 117-129 (2018).

11. Vasu, S., et al. MicroRNA Signatures as Future Biomarkers for Diagnosis of Diabetes States. Cells 8(2019).

12. Safdar, A., Saleem, A. \& Tarnopolsky, M.A. The potential of endurance exercise-derived exosomes to treat metabolic diseases. Nat Rev Endocrinol 12, 504-517 (2016). 
13. Fruhbeis, C., Helmig, S., Tug, S., Simon, P. \& Kramer-Albers, E.M. Physical exercise induces rapid release of small extracellular vesicles into the circulation. Journal of extracellular vesicles 4, 28239 (2015).

14. Whitham, M., et al. Extracellular Vesicles Provide a Means for Tissue Crosstalk during Exercise. Cell metabolism 27, 237-251 e234 (2018).

15. Mulcahy, L.A., Pink, R.C. \& Carter, D.R. Routes and mechanisms of extracellular vesicle uptake. Journal of extracellular vesicles $\mathbf{3}(2014)$.

16. Roden, M. \& Shulman, G.I. The integrative biology of type 2 diabetes. Nature 576, 51-60 (2019).

17. Welton, J.L., Webber, J.P., Botos, L.A., Jones, M. \& Clayton, A. Ready-made chromatography columns for extracellular vesicle isolation from plasma. Journal of extracellular vesicles 4, 27269 (2015).

18. Tam, C.S., et al. Defining insulin resistance from hyperinsulinemic-euglycemic clamps. Diabetes care 35, 1605-1610 (2012).

19. Larsen, S., et al. Biomarkers of mitochondrial content in skeletal muscle of healthy young human subjects. The Journal of physiology 590, 3349-3360 (2012).

20. Gancheva, S., Jelenik, T., Alvarez-Hernandez, E. \& Roden, M. Interorgan Metabolic Crosstalk in Human Insulin Resistance. Physiological reviews 98, 1371-1415 (2018).

21. Shoelson, S.E., Lee, J. \& Yuan, M. Inflammation and the IKK beta/I kappa B/NF-kappa B axis in obesity- and diet-induced insulin resistance. Int J Obes Relat Metab Disord 27 Suppl 3, S49-52 (2003).

22. Powers, S.K., Talbert, E.E. \& Adhihetty, P.J. Reactive oxygen and nitrogen species as intracellular signals in skeletal muscle. The Journal of physiology 589, 2129-2138 (2011).

23. Mathivanan, S., Ji, H. \& Simpson, R.J. Exosomes: extracellular organelles important in intercellular communication. J Proteomics 73, 1907-1920 (2010).

24. Kurgan, N., et al. Changes to the Human Serum Proteome in Response to High Intensity Interval Exercise: A Sequential Top-Down Proteomic Analysis. Front Physiol 10, 362 (2019).

25. Barazzoni, R., et al. Insulin acutely increases fibrinogen production in individuals with type 2 diabetes but not in individuals without diabetes. Diabetes 52, 1851-1856 (2003).

26. Saccani, S., Marazzi, I., Beg, A.A. \& Natoli, G. Degradation of promoter-bound p65/RelA is essential for the prompt termination of the nuclear factor kappaB response. J Exp Med 200, 107-113 (2004).

27. Collins, P.E., Mitxitorena, I. \& Carmody, R.J. The Ubiquitination of NF-kappaB Subunits in the Control of Transcription. Cells $\mathbf{5}(2016)$.

28. Stanton, R.C. Glucose-6-phosphate dehydrogenase, NADPH, and cell survival. IUBMB Life 64, 362369 (2012).

29. Perez-Lopez, A., et al. Antioxidants Facilitate High-intensity Exercise IL-15 Expression in Skeletal Muscle. Int J Sports Med 40, 16-22 (2019).

30. Messenger, S.W., Woo, S.S., Sun, Z. \& Martin, T.F.J. A Ca(2+)-stimulated exosome release pathway in cancer cells is regulated by Munc13-4. J Cell Bio/ 217, 2877-2890 (2018). 
31. Wu, H. \& Ballantyne, C.M. Skeletal muscle inflammation and insulin resistance in obesity. J Clin Invest 127, 43-54 (2017).

32. Little, J.P., et al. Low-volume high-intensity interval training reduces hyperglycemia and increases muscle mitochondrial capacity in patients with type 2 diabetes. Journal of applied physiology (Bethesda, Md. : 1985) 111, 1554-1560 (2011).

33. Toledo, F.G., et al. Effects of physical activity and weight loss on skeletal muscle mitochondria and relationship with glucose control in type 2 diabetes. Diabetes 56, 2142-2147 (2007).

34. Pesta, D. \& Roden, M. The Janus Head of Oxidative Stress in Metabolic Diseases and During Physical Exercise. Curr Diab Rep 17, 41 (2017).

35. Cassidy, S., Thoma, C., Houghton, D. \& Trenell, M.I. High-intensity interval training: a review of its impact on glucose control and cardiometabolic health. Diabetologia 60, 7-23 (2017).

36. Matos, M.A.d., et al. High-Intensity Interval Training Improves Markers of Oxidative Metabolism in Skeletal Muscle of Individuals With Obesity and Insulin Resistance. Frontiers in Physiology 9(2018).

37. Perseghin, G., et al. Increased glucose transport-phosphorylation and muscle glycogen synthesis after exercise training in insulin-resistant subjects. N Engl J Med 335, 1357-1362 (1996).

38. Taylor, R., et al. Remission of Human Type 2 Diabetes Requires Decrease in Liver and Pancreas Fat Content but Is Dependent upon Capacity for beta Cell Recovery. Cell metabolism 28, 667 (2018).

39. Pickering, C. \& Kiely, J. Exercise genetics: seeking clarity from noise. BMJ Open Sport Exerc Med 3, e000309 (2017).

40. An, P., et al. Genome-wide linkage scans for prediabetes phenotypes in response to 20 weeks of endurance exercise training in non-diabetic whites and blacks: the HERITAGE Family Study. Diabetologia 48, 1142-1149 (2005).

41. Kacerovsky-Bielesz, G., et al. Short-term exercise training does not stimulate skeletal muscle ATP synthesis in relatives of humans with type 2 diabetes. Diabetes 58, 1333-1341 (2009).

42. Stephens, N.A., et al. Exercise Response Variations in Skeletal Muscle PCr Recovery Rate and Insulin Sensitivity Relate to Muscle Epigenomic Profiles in Individuals With Type 2 Diabetes. Diabetes care 41, 2245-2254 (2018).

43. Szendroedi, J., et al. Role of diacylglycerol activation of PKCtheta in lipid-induced muscle insulin resistance in humans. Proceedings of the National Academy of Sciences of the United States of America 111, 9597-9602 (2014).

44. Brouwers, B., et al. Exercise training reduces intrahepatic lipid content in people with and people without nonalcoholic fatty liver. American journal of physiology. Endocrinology and metabolism 314, E165-E173 (2018).

45. Freeman, D.W., et al. Altered Extracellular Vesicle Concentration, Cargo, and Function in Diabetes. Diabetes 67, 2377-2388 (2018).

46. Amosse, J., et al. Phenotyping of circulating extracellular vesicles (EVs) in obesity identifies large EVs as functional conveyors of Macrophage Migration Inhibitory Factor. Mol Metab 18, 134-142 (2018). 
47. Pedersen, B.K. Muscle as a secretory organ. Compr Physio/ 3, 1337-1362 (2013).

48. Arkun, Y. Dynamic Modeling and Analysis of the Cross-Talk between Insulin/AKT and MAPK/ERK Signaling Pathways. PloS one 11, e0149684 (2016).

49. Schmitz-Peiffer, C., et al. Inhibition of PKCepsilon improves glucose-stimulated insulin secretion and reduces insulin clearance. Cell metabolism 6, 320-328 (2007).

50. He, L., et al. Activation of the cAMP-PKA pathway Antagonizes Metformin Suppression of Hepatic Glucose Production. J Biol Chem 291, 10562-10570 (2016).

51. Bloch-Damti, A., et al. Differential effects of IRS1 phosphorylated on Ser307 or Ser632 in the induction of insulin resistance by oxidative stress. Diabetologia 49, 2463-2473 (2006).

52. Cai, D., et al. Local and systemic insulin resistance resulting from hepatic activation of IKK-beta and NF-kappaB. Nat Med 11, 183-190 (2005).

53. Vainshtein, A. \& Hood, D.A. The regulation of autophagy during exercise in skeletal muscle. Journal of applied physiology (Bethesda, Md. : 1985) 120, 664-673 (2016).

54. Szendroedi, J., et al. Cohort profile: the German Diabetes Study (GDS). Cardiovascular diabetology 15, 59 (2016).

55. Apostolopoulou, M., et al. Metabolic flexibility and oxidative capacity independently associate with insulin sensitivity in individuals with newly diagnosed type 2 diabetes. Diabetologia 59, 2203-2207 (2016).

56. Kahl, S., et al. Empagliflozin Effectively Lowers Liver Fat Content in Well-Controlled Type 2 Diabetes: A Randomized, Double-Blind, Phase 4, Placebo-Controlled Trial. Diabetes care (2019).

57. Livingstone, R.S., et al. Initial clinical application of modified Dixon with flexible echo times: hepatic and pancreatic fat assessments in comparison with (1)H MRS. MAGMA 27, 397-405 (2014).

58. Morgunov, I. \& Srere, P.A. Interaction between citrate synthase and malate dehydrogenase. Substrate channeling of oxaloacetate. J Biol Chem 273, 29540-29544 (1998).

59. Mahmood, T. \& Yang, P.C. Western blot: technique, theory, and trouble shooting. N Am J Med Sci 4, 429-434 (2012).

60. Lambernd, S., et al. Contractile activity of human skeletal muscle cells prevents insulin resistance by inhibiting pro-inflammatory signalling pathways. Diabetologia 55, 1128-1139 (2012).

61. Hartwig, S., et al. Exosomal proteins constitute an essential part of the human adipose tissue secretome. Biochim Biophys Acta Proteins Proteom 1867, 140172 (2019).

62. Lobb, R.J., et al. Optimized exosome isolation protocol for cell culture supernatant and human plasma. Journal of extracellular vesicles 4, 27031 (2015).

63. Ducret, A., Van Oostveen, I., Eng, J.K., Yates, J.R., 3rd \& Aebersold, R. High throughput protein characterization by automated reverse-phase chromatography/electrospray tandem mass spectrometry. Protein Sci 7, 706-719 (1998).

64. Bendtsen, J.D., Jensen, L.J., Blom, N., Von Heijne, G. \& Brunak, S. Feature-based prediction of nonclassical and leaderless protein secretion. Protein Eng Des Sel 17, 349-356 (2004). 

analysis tool. Proteomics 15, 2597-2601 (2015).

\section{Tables}

Table 1. Anthropometric and metabolic parameters at baseline and after 12 weeks of HIIT (12-week) in patients with type 2 diabetes (T2D), insulin-resistant controls (IR NDM) and insulin-sensitive controls (IS NDM)

\begin{tabular}{|c|c|c|c|c|c|c|}
\hline \multirow[t]{2}{*}{ Parameter } & \multicolumn{2}{|l|}{$\mathrm{T} 2 \mathrm{D}(\mathrm{n}=20)$} & \multicolumn{2}{|l|}{ IR NDM $(n=11)$} & \multicolumn{2}{|c|}{ IS NDM $(n=12)$} \\
\hline & Baseline & 12-week & Baseline & 12-week & Baseline & 12-week \\
\hline Age (years) & $57 \pm 1$ & & $56 \pm 1$ & & $58 \pm 1$ & \\
\hline BMI (kg/mワ) & $31.2 \pm 0.6 \&$ & $30.8 \pm 0.6$ & $32.2 \pm 0.7$ & $32.1 \pm 0.7$ & $29.3 \pm 0.6$ \#\# & $29.1 \pm 0.4$ \\
\hline Whole-body fat (kg) & $26.5 \pm 1.1$ & $26.5 \pm 1.4$ & $29.6 \pm 1.9$ & $29.1 \pm 2.1$ & $23.9 \pm 1.4{ }^{\#}$ & $23.1 \pm 1.4$ \\
\hline Visceral fat (kg) & $5.9 \pm 0.4 \& \&$ & $5.5 \pm 0.4 *$ & $5.4 \pm 0.4$ & $5.4 \pm 0.4$ & $4.4 \pm 0.2^{\#}$ & $4.1 \pm 0.2$ \\
\hline Subcutaneous fat (kg) & $20.6 \pm 1.1$ & $20.9 \pm 1.3$ & $24.2 \pm 1.8$ & $23.7 \pm 1.9$ & $19.5 \pm 1.4^{\#}$ & $18.9 \pm 1,3$ \\
\hline HbA1c (\%) & $7.2 \pm 0.2 \& \& \& \& \S \S \S$ & $6.9 \pm 0.2$ & $5.4 \pm 0.1$ & $5.3 \pm 0.1$ & $5.4 \pm 0.1$ & $5.5 \pm 0.1$ \\
\hline SGPT (U/l) & $41(29,62) \& \&$ & $33(25,61)$ & $29(20,37)$ & $27(19,32)$ & $24(21,29)$ & $24(20,29)$ \\
\hline Free fatty acids $(\mu \mathrm{mol} / \mathrm{l})$ & $523 \pm 38$ & $369 \pm 26^{* * *}$ & $504 \pm 59$ & $463 \pm 44$ & $415 \pm 50$ & $411 \pm 59$ \\
\hline Triglycerides (mg/dl) & $152(125,231)$ & $119(90,173) * *$ & $131(102,163)$ & $132(100,143)$ & $121(82,149)$ & $128(81,160)$ \\
\hline LDL-cholesterol (mg/dl) & $132 \pm 8^{\S}$ & $128 \pm 7$ & $158 \pm 9$ & $153 \pm 12$ & $149 \pm 7$ & $133 \pm 9$ \\
\hline HDL-cholesterol (mg/dl) & $46 \pm 3^{\S \&}$ & $51 \pm 3 *$ & $55 \pm 3$ & $56 \pm 3$ & $55 \pm 4$ & $50 \pm 4 *$ \\
\hline
\end{tabular}

BMI: body mass index, HbA1c: hemoglobin A1c, SGPT: serum glutamic-pyruvic transaminase, LDL: low-density-lipoprotein, HDL: high-density-lipoprotein. Data are shown as mean \pm SEM or median $(\mathrm{q} 1, \mathrm{q} 3), \&_{\mathrm{p}}<0.05, \& \& \mathrm{p}<0.01, \& \& \& \mathrm{p}<0.001$ T2D vs IS NDM, $\S \mathrm{p}<0.05$ T2D vs IR NDM, ${ }^{*} \mathrm{p}<0.05,{ }^{\#} \mathrm{p}<0.01,{ }^{*}{ }^{*} \mathrm{p}<0.001$ IS NDM vs IR NDM, ${ }^{*} \mathrm{p}<0.05$, $* * \mathrm{p}<0.001, * * * \mathrm{p}<0.0000112$-week vs. baseline

\section{Figures}


(a)

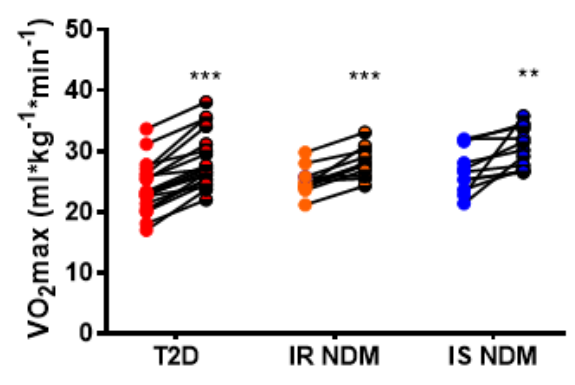

(c)

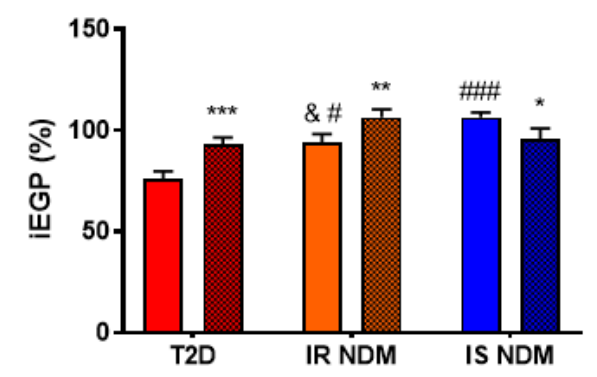

(e)

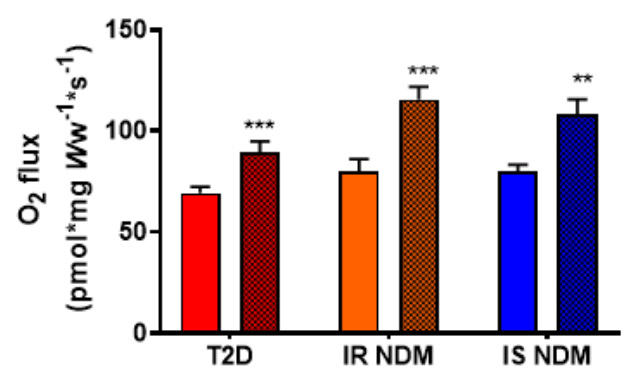

(g)

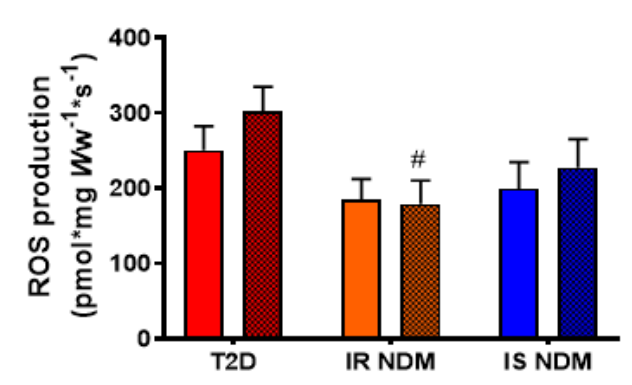

(b)

baseline

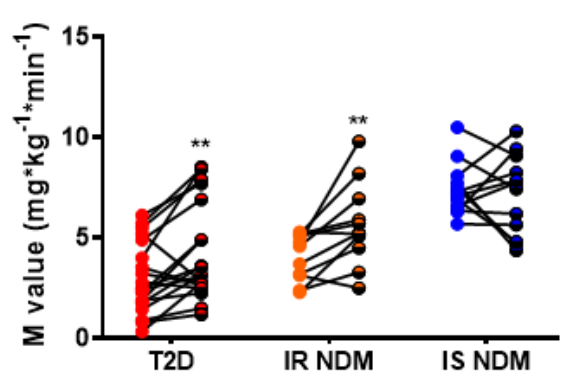

(d)

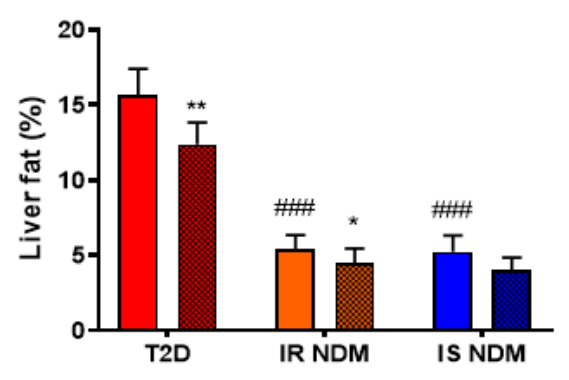

(f)

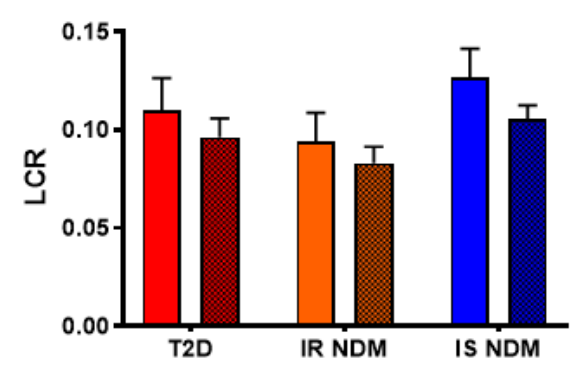

(h)

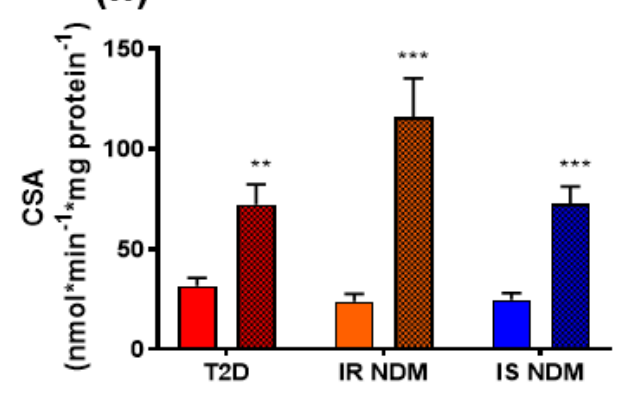

\section{Figure 1}

HIIT induces metabolic changes and stimulates maximal skeletal muscle mitochondrial capacity in humans with (T2D) and without type 2 diabetes (NDM) (a) Maximal oxygen uptake (VO2max) (*** $p=0.00025$ for T2D, ${ }^{* \star *} p=6.42 \times 10-5$ for IR NDM, ${ }^{* \star} p=0.008$ for IS NDM) , (b) peripheral insulin sensitivity (M-value) ( ${ }^{*} p=0.003$ for T2D, $* \star p=0.002$ for IR NDM), (c) hepatic insulin sensitivity (suppression of endogenous glucose production, iEGP) during high-insulin clamp (*** $p=0.0001$ for T2D, 
$\star \star p=0.007$ for IR NDM, * $p=0.02$ for IS NDM; \#p=0.01 T2D vs IR NDM, \#\#\#p=7.87x10-7 T2D vs IS NDM; $\& p=0.04$ IR NDM vs IS NDM) (d) liver fat content (** $p=0.003$ for T2D, ${ }^{*}=0.02$ for IR NDM; \#\#\#p=0.0005 T2D vs IR NDM, \#\#\#p=7.17x10-5 T2D vs IS NDM), (e) maximal uncoupled respiration ( $W w=$ wet weight) (*** $p=3.73 \times 10-5$ for T2D, ${ }^{* \star *} p=0.0005$ for IR NDM, ${ }^{\star *} p=0.001$ for IS NDM), (f) leak control ratio (LCR), (g) reactive oxygen species (ROS) emission (\#p=0.03 T2D vs IR NDM) and ( $h$ ) citrate synthase activity (CSA) (** $p=0.004$ for T2D, ${ }^{* \star} p=0.0006$ for IR NDM, ${ }^{* \star *} p=0.0003$ for IS NDM) at baseline and after 12-week HIIT in persons with T2D, IR NDM and IS NDM. Data are presented as mean \pm standard error of mean (SEM).

(a)

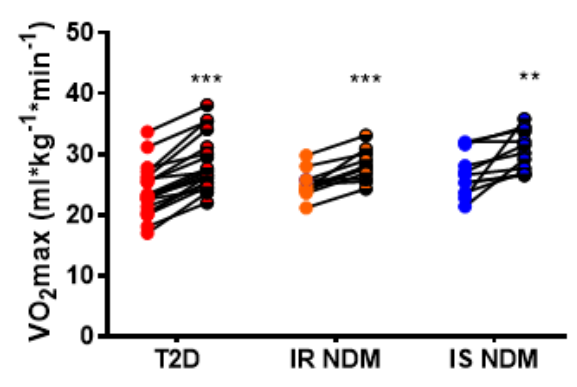

(c)

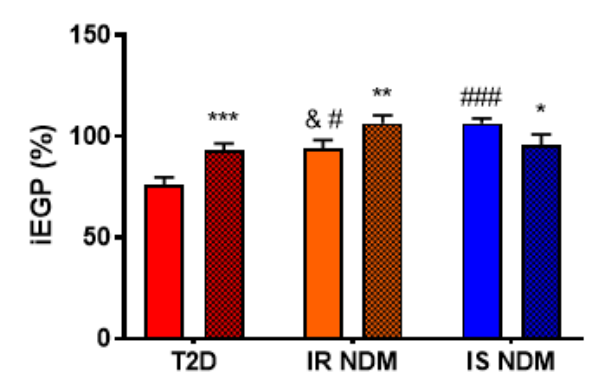

(e)

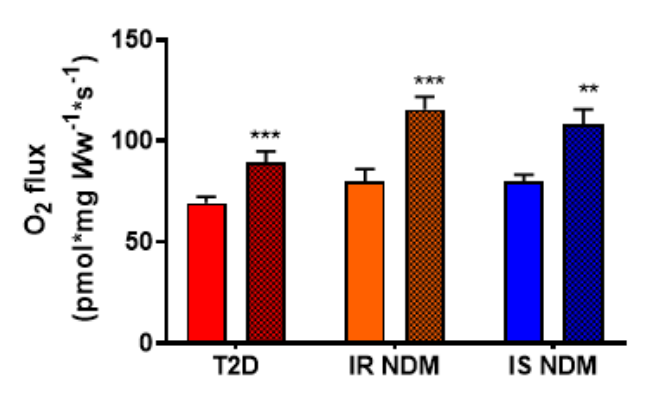

(g)

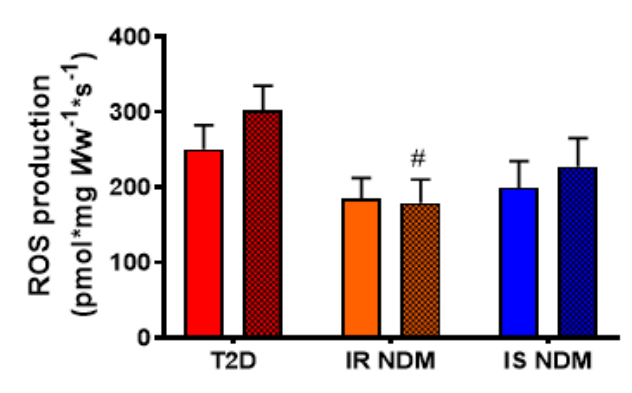

(b)

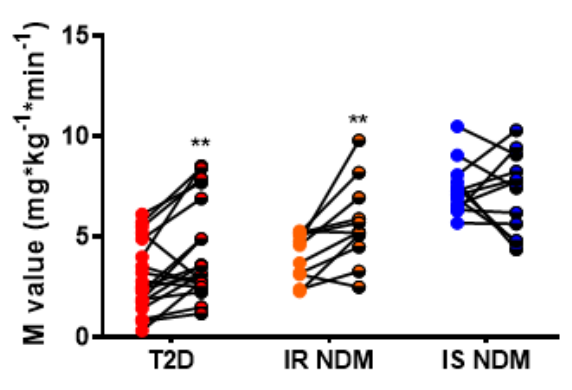

(d)

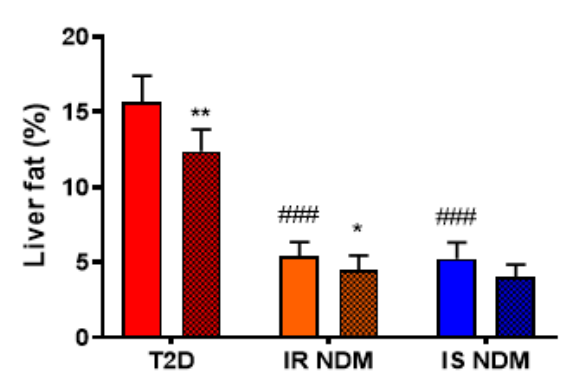

(f)

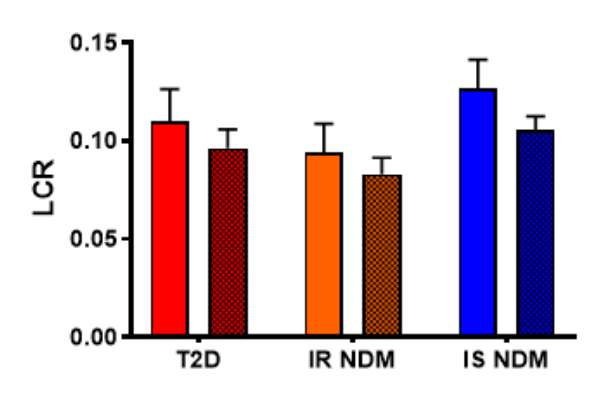

(h)

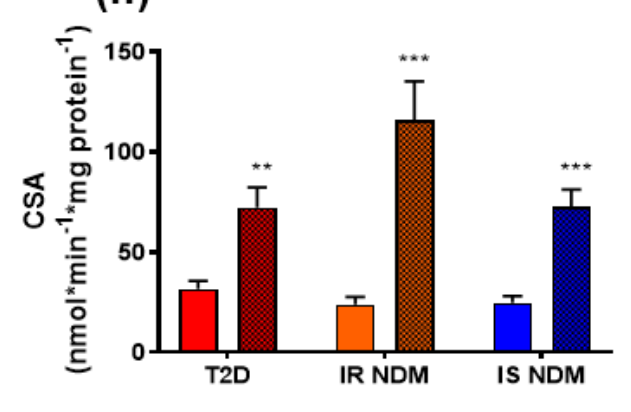




\section{Figure 1}

HIIT induces metabolic changes and stimulates maximal skeletal muscle mitochondrial capacity in humans with (T2D) and without type 2 diabetes (NDM) (a) Maximal oxygen uptake (VO2max)

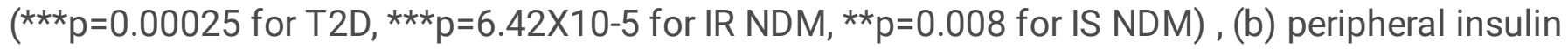
sensitivity (M-value) ( ${ }^{\star *} p=0.003$ for T2D, ${ }^{* \star} p=0.002$ for IR NDM), (c) hepatic insulin sensitivity (suppression of endogenous glucose production, iEGP) during high-insulin clamp ( ${ }^{\star \star \star} p=0.0001$ for T2D, ${ }^{* \star} p=0.007$ for IR NDM, ${ }^{*} p=0.02$ for IS NDM; \#p=0.01 T2D vs IR NDM, \#\#\#p=7.87x10-7 T2D vs IS NDM; $\& p=0.04$ IR NDM vs IS NDM) (d) liver fat content (** $p=0.003$ for T2D, ${ }^{*} p=0.02$ for IR NDM; \#\#\#p=0.0005 T2D vs IR NDM, \#\#\#p=7.17x10-5 T2D vs IS NDM), (e) maximal uncoupled respiration (Ww= wet weight) ( ${ }^{\star \star *} p=3.73 \times 10-5$ for T2D, ${ }^{\star * \star} p=0.0005$ for IR NDM, ${ }^{\star \star} p=0.001$ for IS NDM), (f) leak control ratio (LCR), (g) reactive oxygen species (ROS) emission (\#p=0.03 T2D vs IR NDM) and ( $h$ ) citrate synthase activity (CSA) ( ${ }^{\star *} p=0.004$ for T2D, ${ }^{* \star *} p=0.0006$ for IR NDM, ${ }^{\star \star *} p=0.0003$ for IS NDM) at baseline and after 12-week HIIT in persons with T2D, IR NDM and IS NDM. Data are presented as mean \pm standard error of mean (SEM). 
(a)

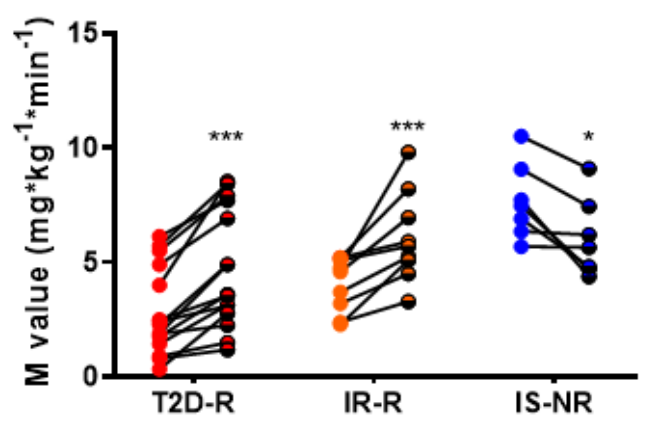

(c)

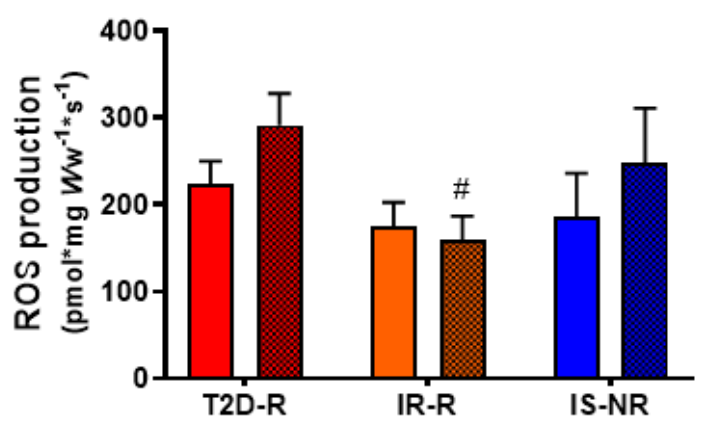

(e)

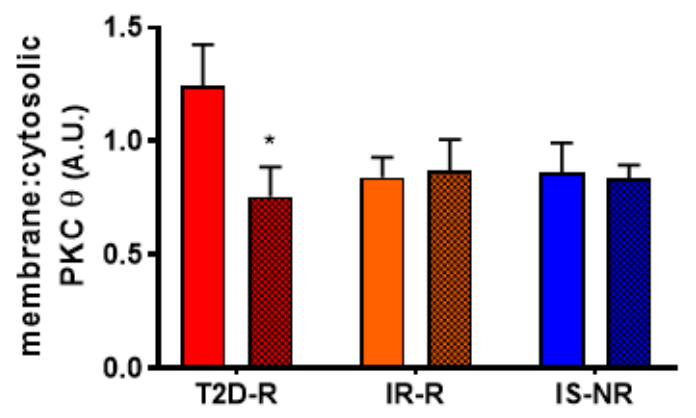

(b)

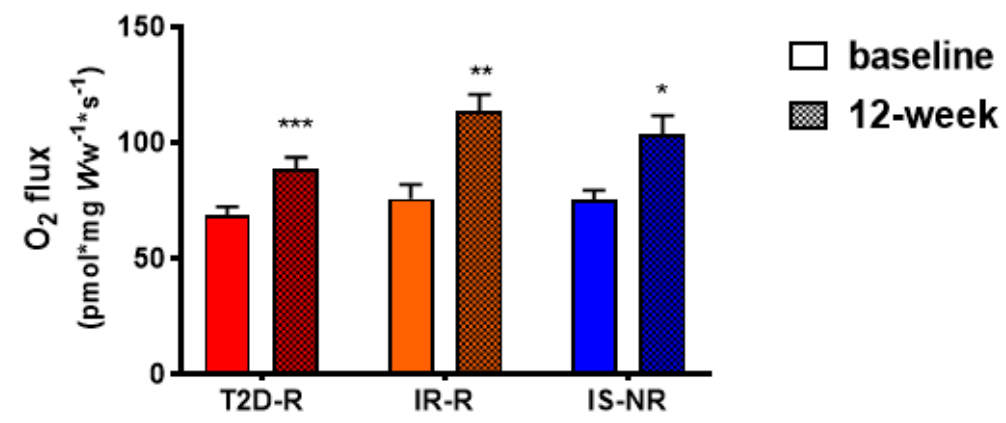

(d)

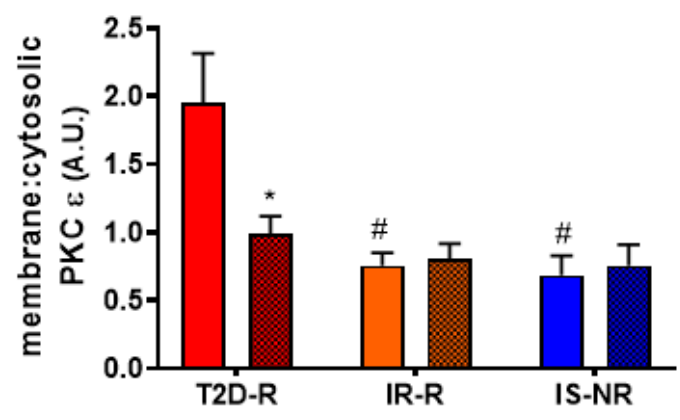

(f)

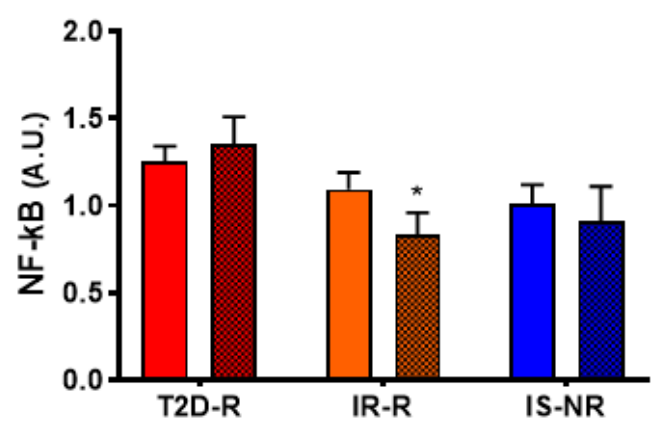

Figure 2

HIIT differently affects myocellular pathways of insulin sensitivity in the insulin-resistant responders

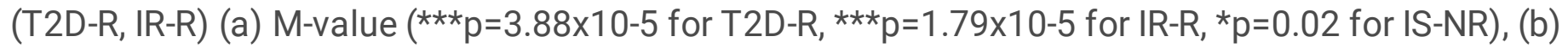
maximal uncoupled respiration ( ${ }^{\star \star \star} p=0.0002$ for T2D-R, ${ }^{\star \star} p=0.002$ for IR-R, ${ }^{\star} p=0.02$ for IS-NR), (c) ROS emission ( $\# p=0.04$ T2D-R vs IR-R), (d) PKC $\varepsilon$ ( ${ }^{*} p=0.02$ for T2D-R; \#=p=0.02 T2D-R vs IR-R, \#p=0.04 T2D-R vs IS-NR), (e) PKC $\theta$ ( ${ }^{*} p=0.03$ for T2D-R) and (f) NF-KB ( ${ }^{*} p=0.01$ for IR-R) western blot analysis in the subgroups T2D-R $(\mathrm{N}=16), \mathrm{IR}-\mathrm{R}(\mathrm{N}=9)$ and IS-NR $(\mathrm{N}=7)$ at baseline and after 12-week HIIT. Data are expressed as mean \pm SEM. 
(a)

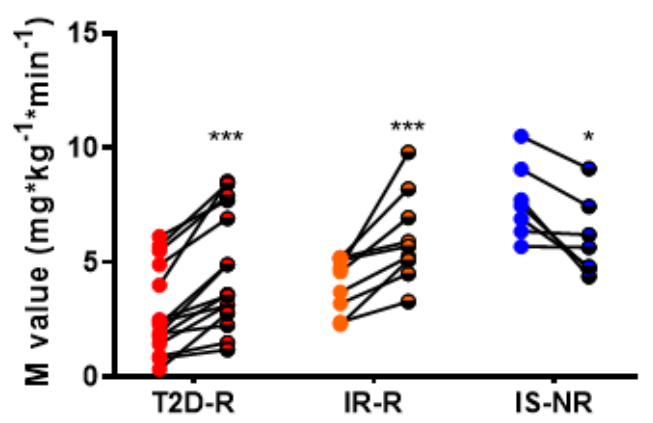

(c)

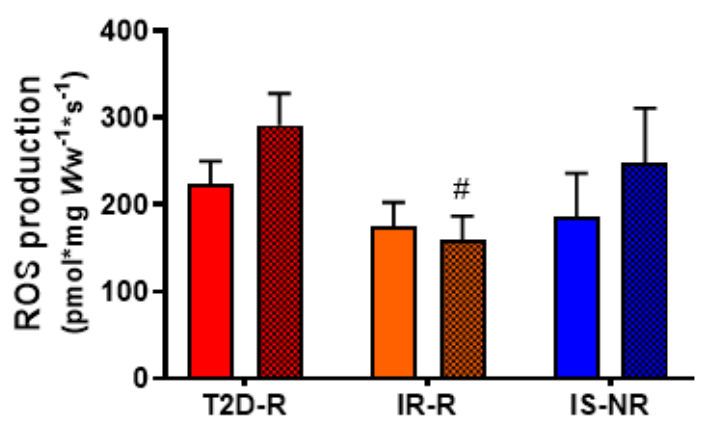

(e)

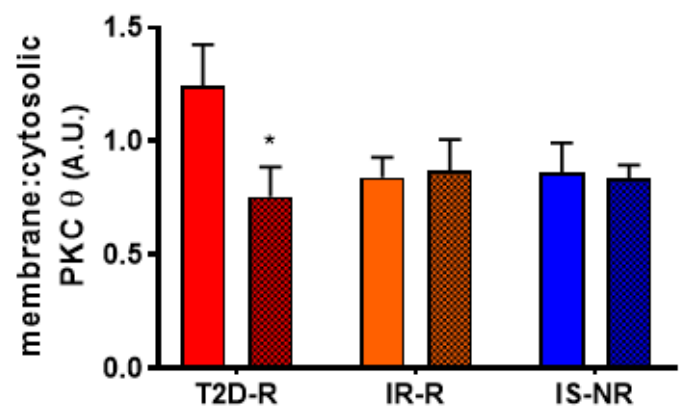

(b)

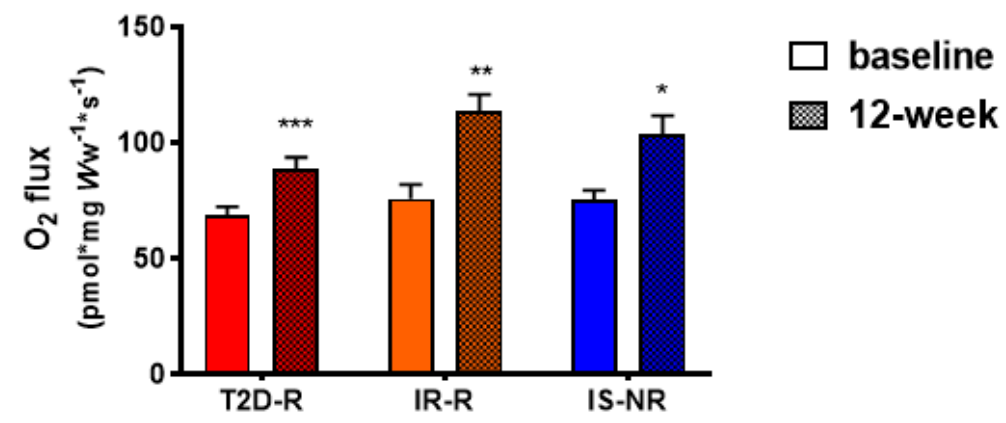

(d)

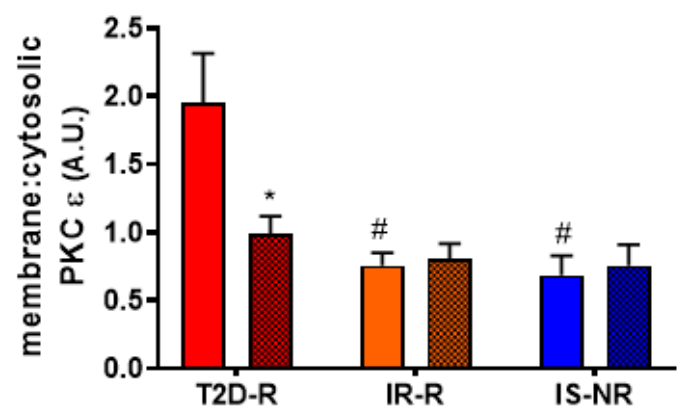

(f)

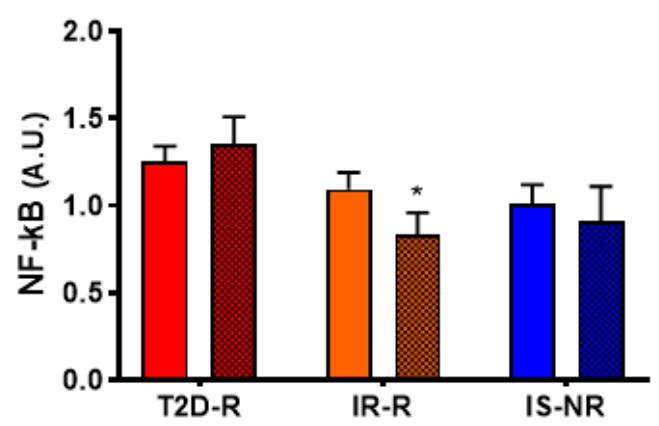

Figure 2

HIIT differently affects myocellular pathways of insulin sensitivity in the insulin-resistant responders

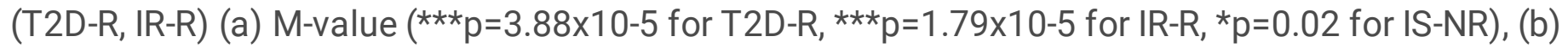
maximal uncoupled respiration ( ${ }^{\star \star \star} p=0.0002$ for T2D-R, ${ }^{\star \star} p=0.002$ for IR-R, ${ }^{\star} p=0.02$ for IS-NR), (c) ROS emission ( $\# p=0.04$ T2D-R vs IR-R), (d) PKC $\varepsilon$ ( ${ }^{*} p=0.02$ for T2D-R; \#=p=0.02 T2D-R vs IR-R, \#p=0.04 T2D-R vs IS-NR), (e) PKC $\theta$ ( ${ }^{*} p=0.03$ for T2D-R) and (f) NF-KB ( ${ }^{*} p=0.01$ for IR-R) western blot analysis in the subgroups T2D-R $(\mathrm{N}=16), \mathrm{IR}-\mathrm{R}(\mathrm{N}=9)$ and IS-NR $(\mathrm{N}=7)$ at baseline and after 12-week HIIT. Data are expressed as mean \pm SEM. 
(a)

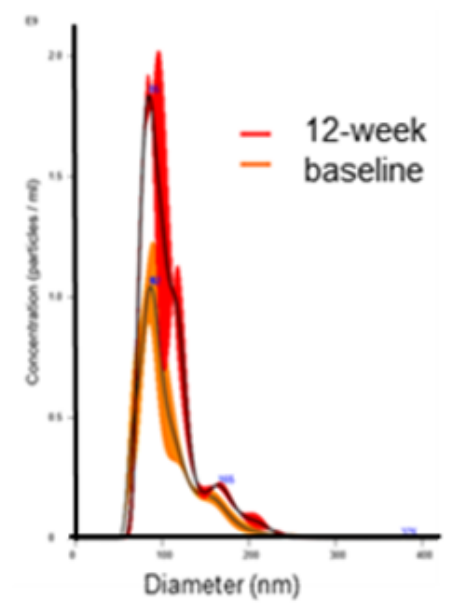

(c)

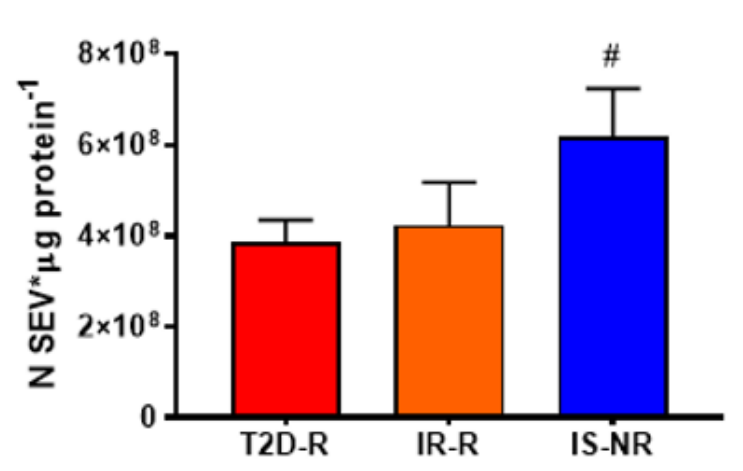

(b)

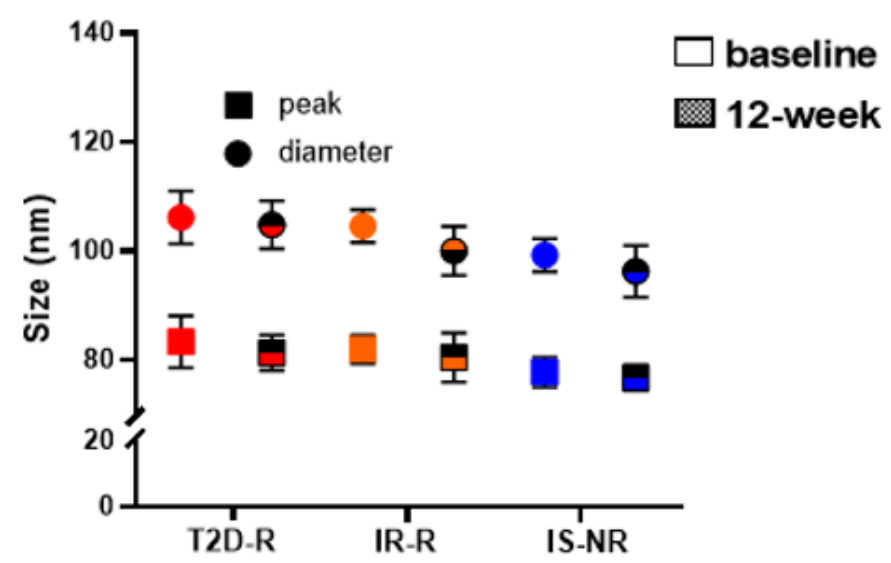

(d)

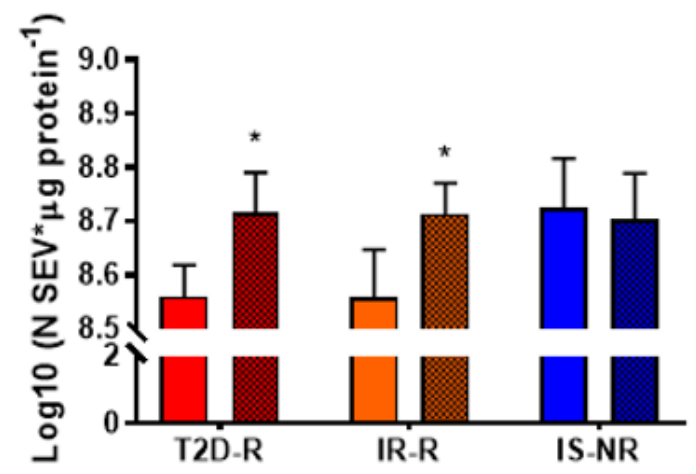

\section{Figure 3}

HIIT intervention increases the release of circulating small extracellular vesicles (SEV) in humans with type 2 diabetes (T2D-R) and insulin-resistant (IR-R) responders, but not in insulin-sensitive non-responders (IS-NR) to exercising (a) Representative distribution profiles of SEV isolated from serum of a T2D patient at baseline (red plot) and after 12-week HIIT (orange plot), (b) plots of peak and diameter sizes of SEV isolated from T2D-R (N=8), IR-R ( $N=8)$ and IS-NR $(N=6)$ at baseline and after 12-wk HIIT, (c) number of circulating SEV at baseline (\#p=0.04 T2D-R vs IS-NR) and (d) number of circulating SEV log10 transformed at baseline and after 12-week HIIT ( ${ }^{*} p=0.01$ for T2D-R, ${ }^{*} p=0.02$ for IR-R) in the pilot group including T2D-R ( $(N=8), I R-R(N=8)$ and IS-NR $(N=6)$ humans. Data are expressed as mean \pm SEM. 
(a)

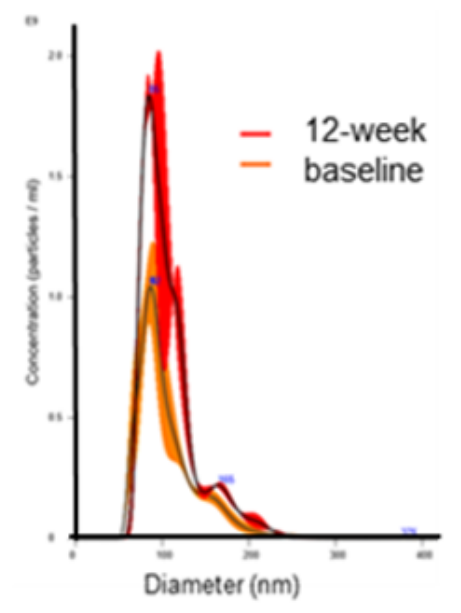

(c)

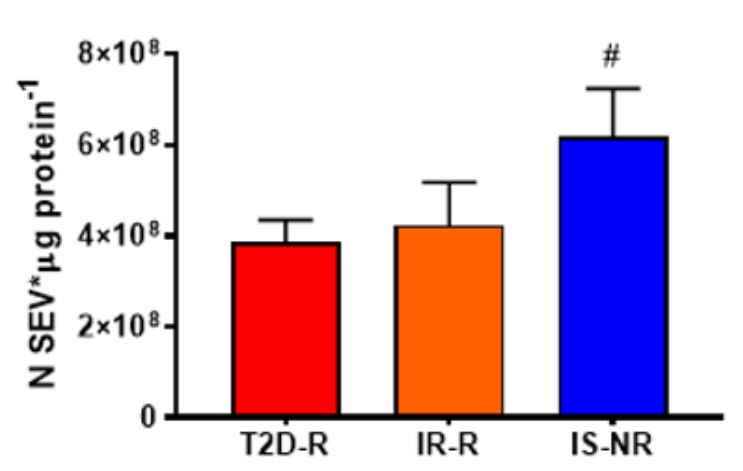

(b)

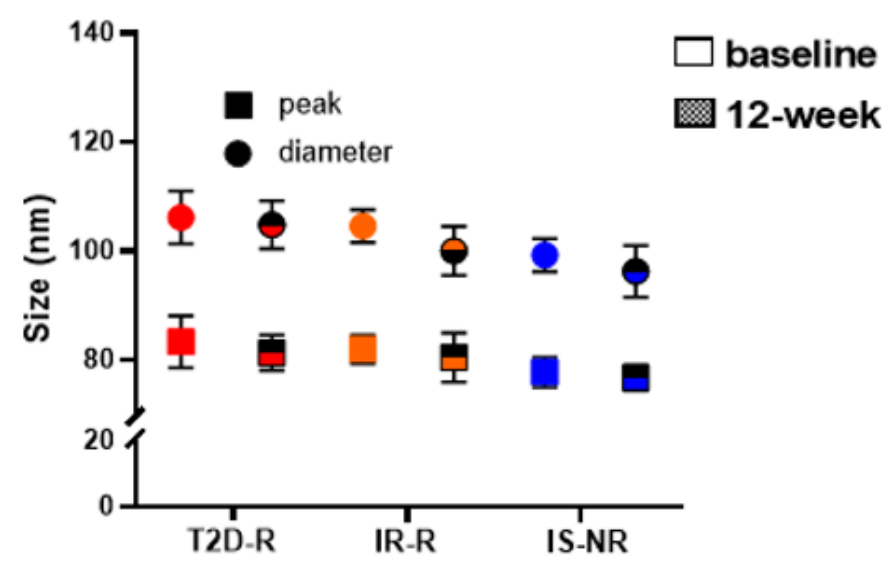

(d)

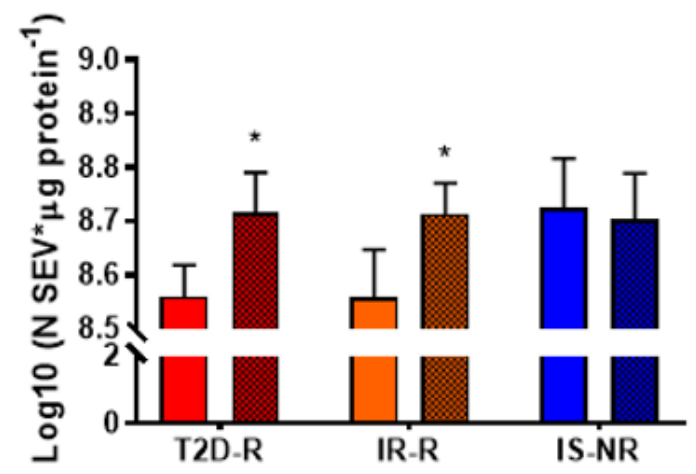

\section{Figure 3}

HIIT intervention increases the release of circulating small extracellular vesicles (SEV) in humans with type 2 diabetes (T2D-R) and insulin-resistant (IR-R) responders, but not in insulin-sensitive non-responders (IS-NR) to exercising (a) Representative distribution profiles of SEV isolated from serum of a T2D patient at baseline (red plot) and after 12-week HIIT (orange plot), (b) plots of peak and diameter sizes of SEV isolated from T2D-R (N=8), IR-R ( $N=8)$ and IS-NR $(N=6)$ at baseline and after 12-wk HIIT, (c) number of circulating SEV at baseline (\#p=0.04 T2D-R vs IS-NR) and (d) number of circulating SEV log10 transformed at baseline and after 12-week HIIT ( ${ }^{*} p=0.01$ for T2D-R, ${ }^{*} p=0.02$ for IR-R) in the pilot group including T2D-R ( $(N=8), I R-R(N=8)$ and IS-NR $(N=6)$ humans. Data are expressed as mean \pm SEM. 
(a)

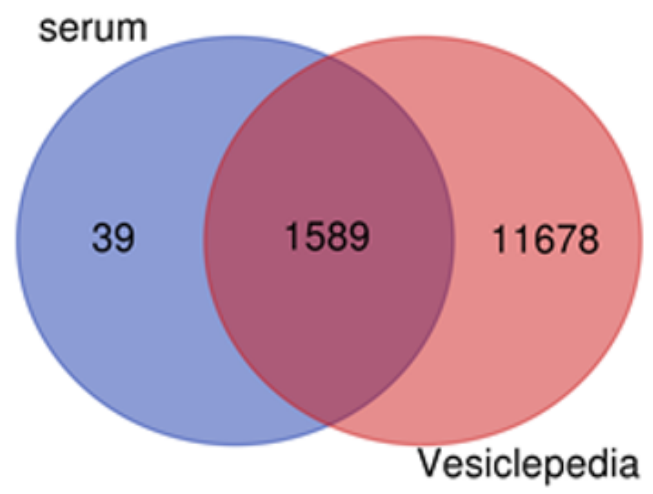

(c)

\section{Cellular component (GO)}

\begin{tabular}{|l|c|c|}
\hline \multicolumn{1}{|c|}{ Pathway description } & p-value & \% of proteins \\
\hline Extracellular exosome & $3.0 \mathrm{E}-116$ & 69.8 \\
Blood microparticle & $2.9 \mathrm{E}-63$ & 20.6 \\
Secretory granule lumen & $4.8 \mathrm{E}-20$ & 9.2 \\
Cytosol & $3.1 \mathrm{E}-18$ & 51.1 \\
Membrane & $3.9 \mathrm{E}-11$ & 25.2 \\
\hline
\end{tabular}

(e)

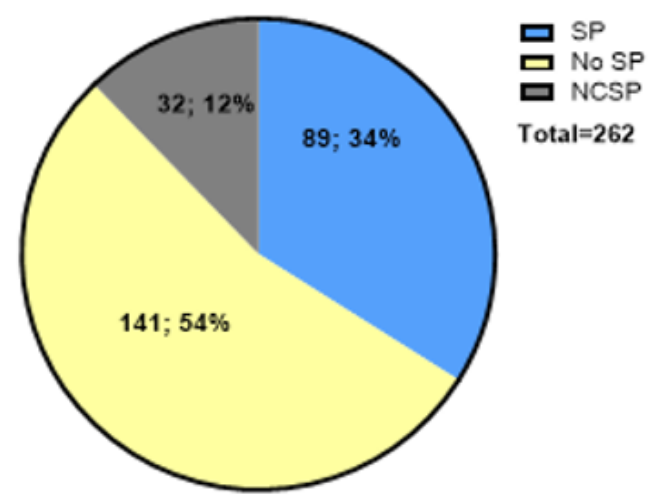

(b)

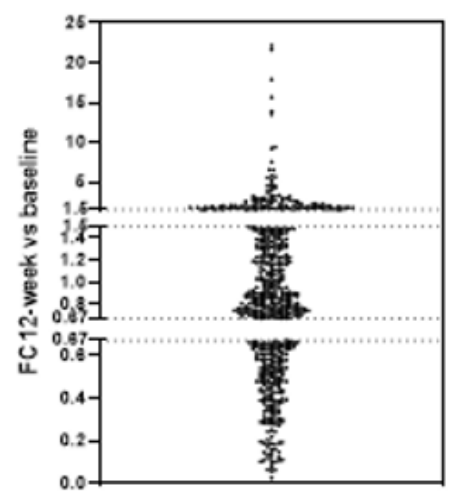

(d)

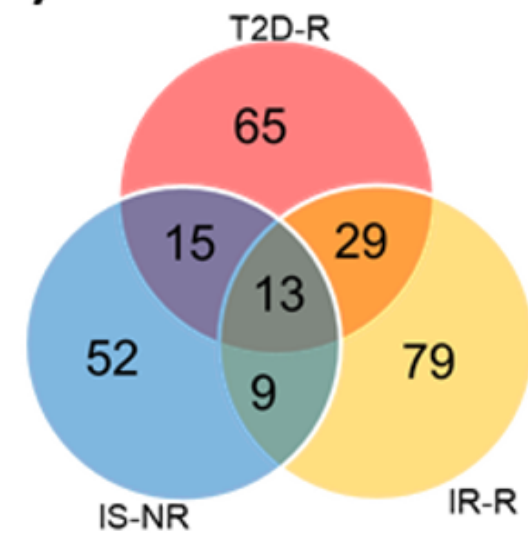

(f)

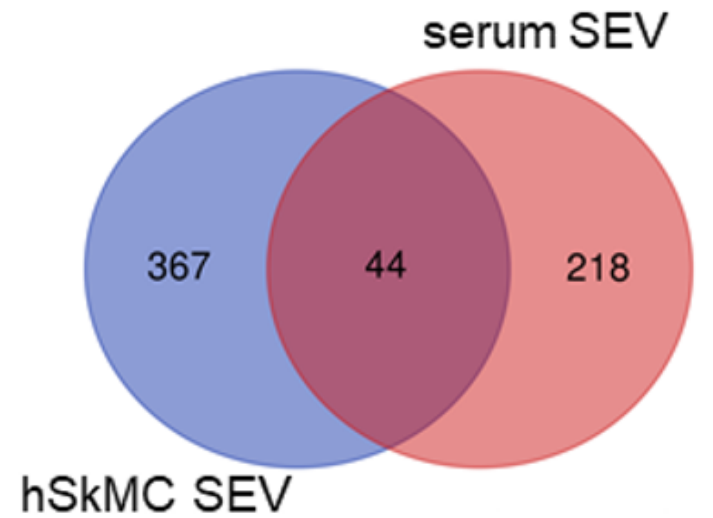

\section{Figure 4}

Characteristics of SEV proteins differentially regulated after 12-week HIIT (a) Venn diagram showing the overlap of the proteins identified in serum SEV with the human protein entries in the EV database Vesiclepedia. Gene products were matched in FunRich. (b) Volcano plot depicting the fold changes in proteins isolated from circulating SEV comparing 12-week HIIT vs baseline: only proteins with a FC $<0.67$ or $>1.5$ (absolute log FC >0.5) were included for further analysis. (c) GO-CC analysis for the $262 \mathrm{SEV}$ 
proteins regulated after 12-week HIIT with p-value and percentage of proteins. (d) Venn diagram showing the overlap of proteins regulated after 12-week HIIT among the three groups. (e) Amino acid sequences of the SEV proteins regulated during exercise (262 entries) were analyzed to predict the presence of a secretory signal peptide (SP). (f) Venn diagram showing the overlap of proteins regulated after 12-week HIIT in circulating SEV and during EPS in hSkMC-derived SEV in vitro.

\section{(a)}

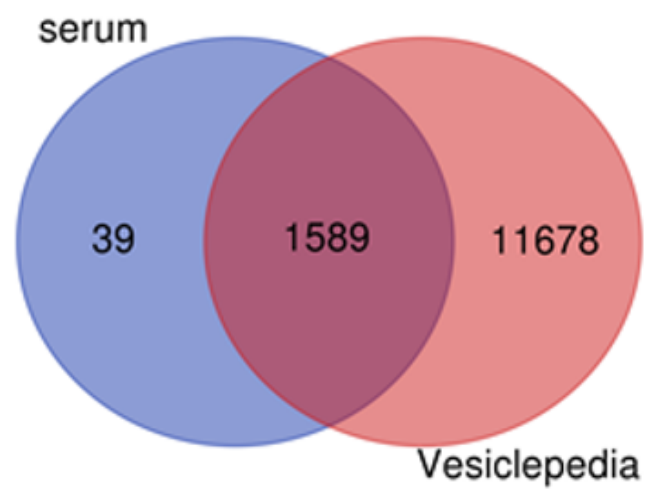

(c)

\section{Cellular component (GO)}

\begin{tabular}{|l|c|c|}
\hline \multicolumn{1}{|c|}{ Pathway description } & p-value & \% of proteins \\
\hline Extracellular exosome & $3.0 \mathrm{E}-116$ & 69.8 \\
Blood microparticle & $2.9 \mathrm{E}-63$ & 20.6 \\
Secretory granule lumen & $4.8 \mathrm{E}-20$ & 9.2 \\
Cytosol & $3.1 \mathrm{E}-18$ & 51.1 \\
Membrane & $3.9 \mathrm{E}-11$ & 25.2 \\
\hline
\end{tabular}

(e)

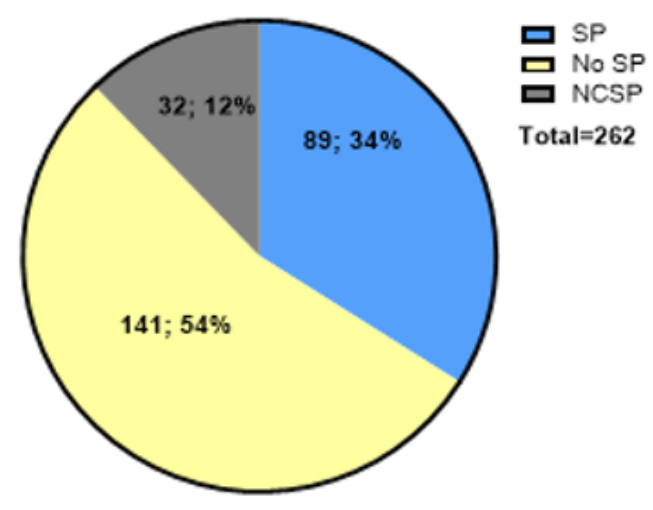

(b)

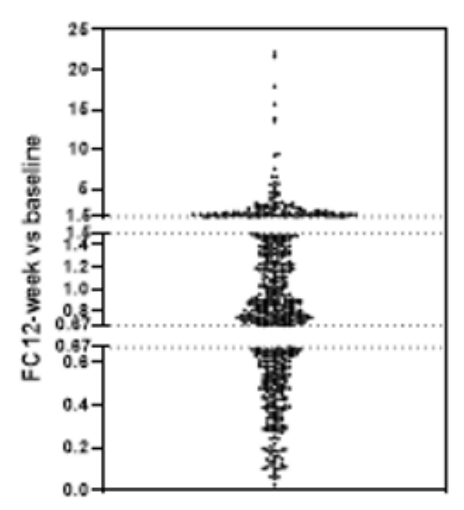

(d)

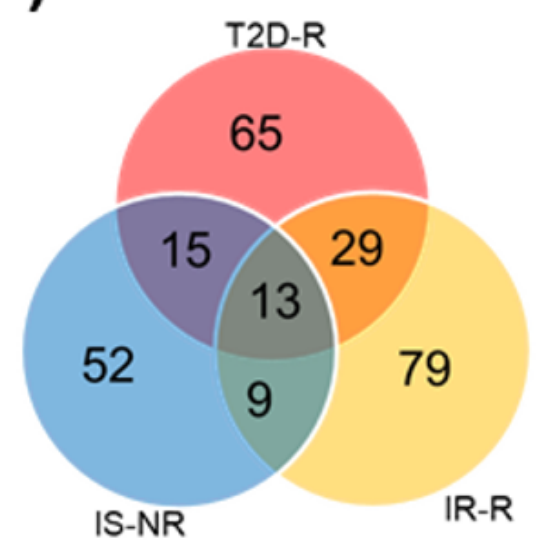

(f)

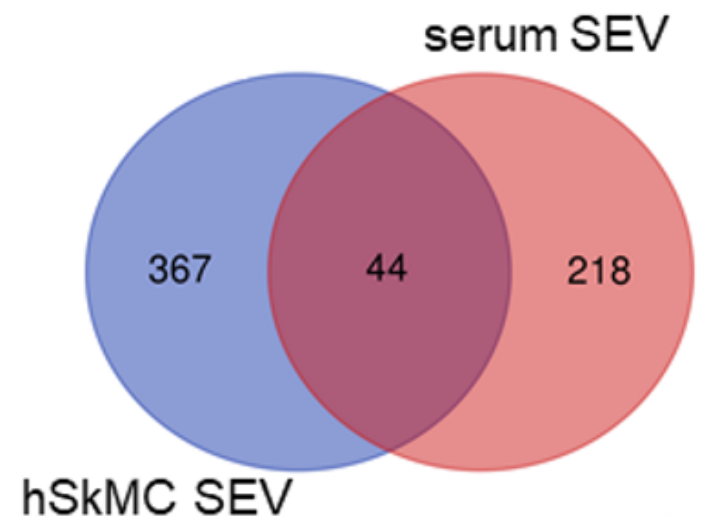

Figure 4 
Characteristics of SEV proteins differentially regulated after 12-week HIIT (a) Venn diagram showing the overlap of the proteins identified in serum SEV with the human protein entries in the EV database Vesiclepedia. Gene products were matched in FunRich. (b) Volcano plot depicting the fold changes in proteins isolated from circulating SEV comparing 12-week HIIT vs baseline: only proteins with a FC $<0.67$ or $>1.5$ (absolute log FC >0.5) were included for further analysis. (c) GO-CC analysis for the $262 \mathrm{SEV}$ proteins regulated after 12-week HIIT with p-value and percentage of proteins. (d) Venn diagram showing the overlap of proteins regulated after 12-week HIIT among the three groups. (e) Amino acid sequences of the SEV proteins regulated during exercise (262 entries) were analyzed to predict the presence of a secretory signal peptide (SP). (f) Venn diagram showing the overlap of proteins regulated after 12-week HIIT in circulating SEV and during EPS in hSkMC-derived SEV in vitro.

(a)

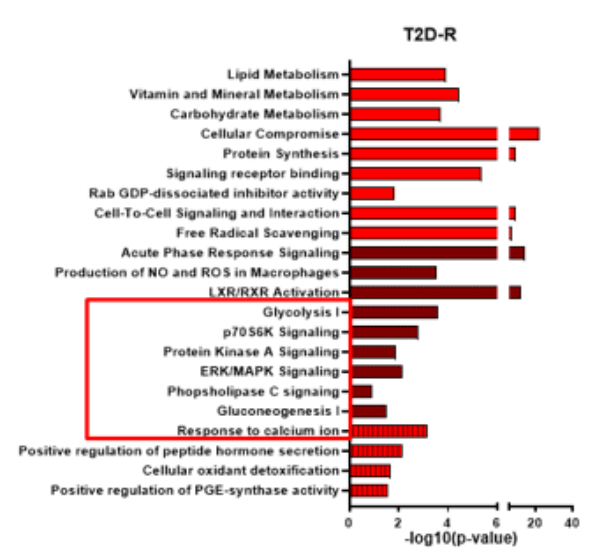

(b)

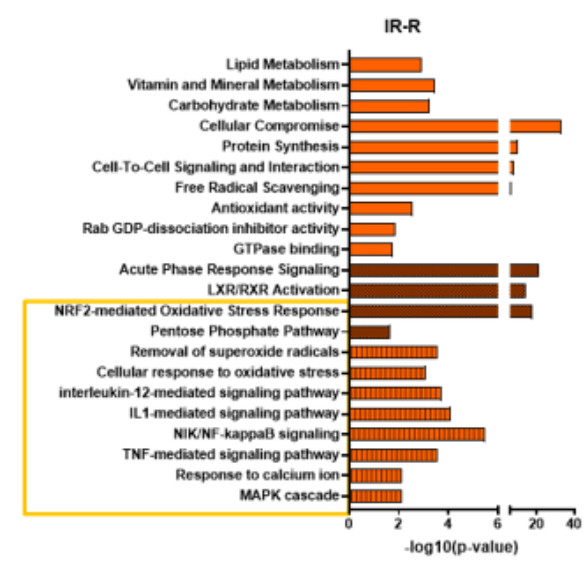

(c)

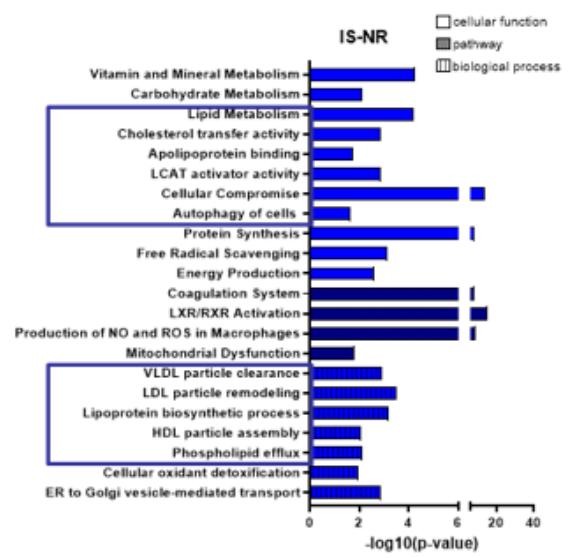

\section{Figure 5}

HIIT differently affects the proteomic profile of small extracellular vesicles (SEV) in insulin-resistant (IR-R and T2D-R) and insulin-sensitive humans Cellular functions, pathways and biological process of the SEV proteins regulated during exercise in T2D-R (a), IR-R (b) and IS-NR (c). The squares indicate the unique pathways for each group.

(a)

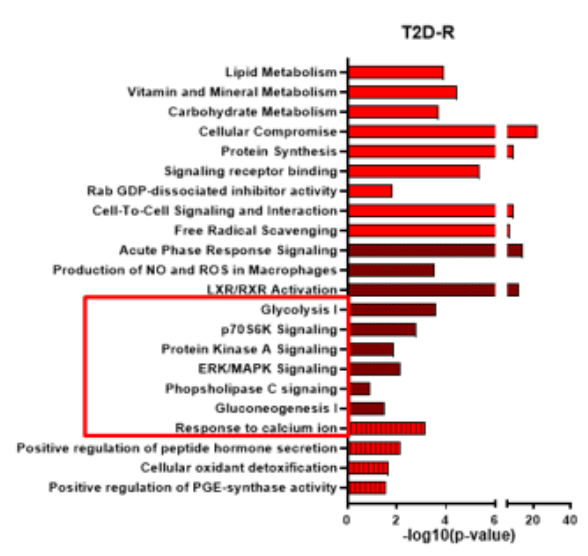

(b)

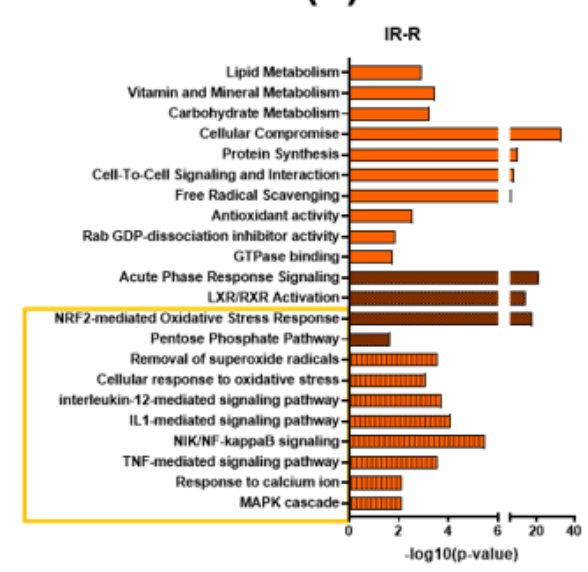

(c)

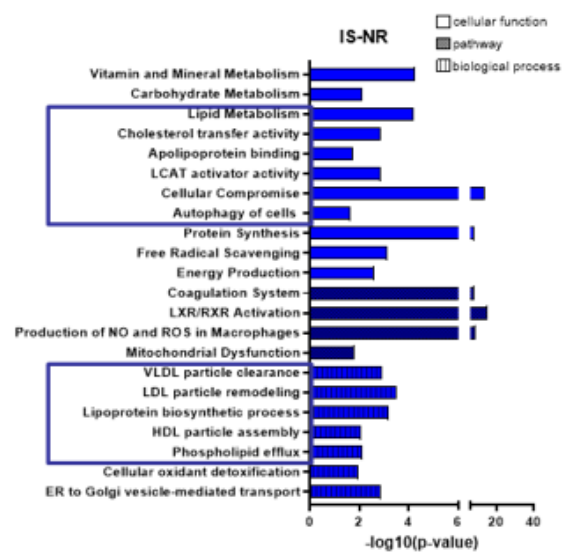


HIIT differently affects the proteomic profile of small extracellular vesicles (SEV) in insulin-resistant (IR-R and T2D-R) and insulin-sensitive humans Cellular functions, pathways and biological process of the SEV proteins regulated during exercise in T2D-R (a), IR-R (b) and IS-NR (c). The squares indicate the unique pathways for each group.
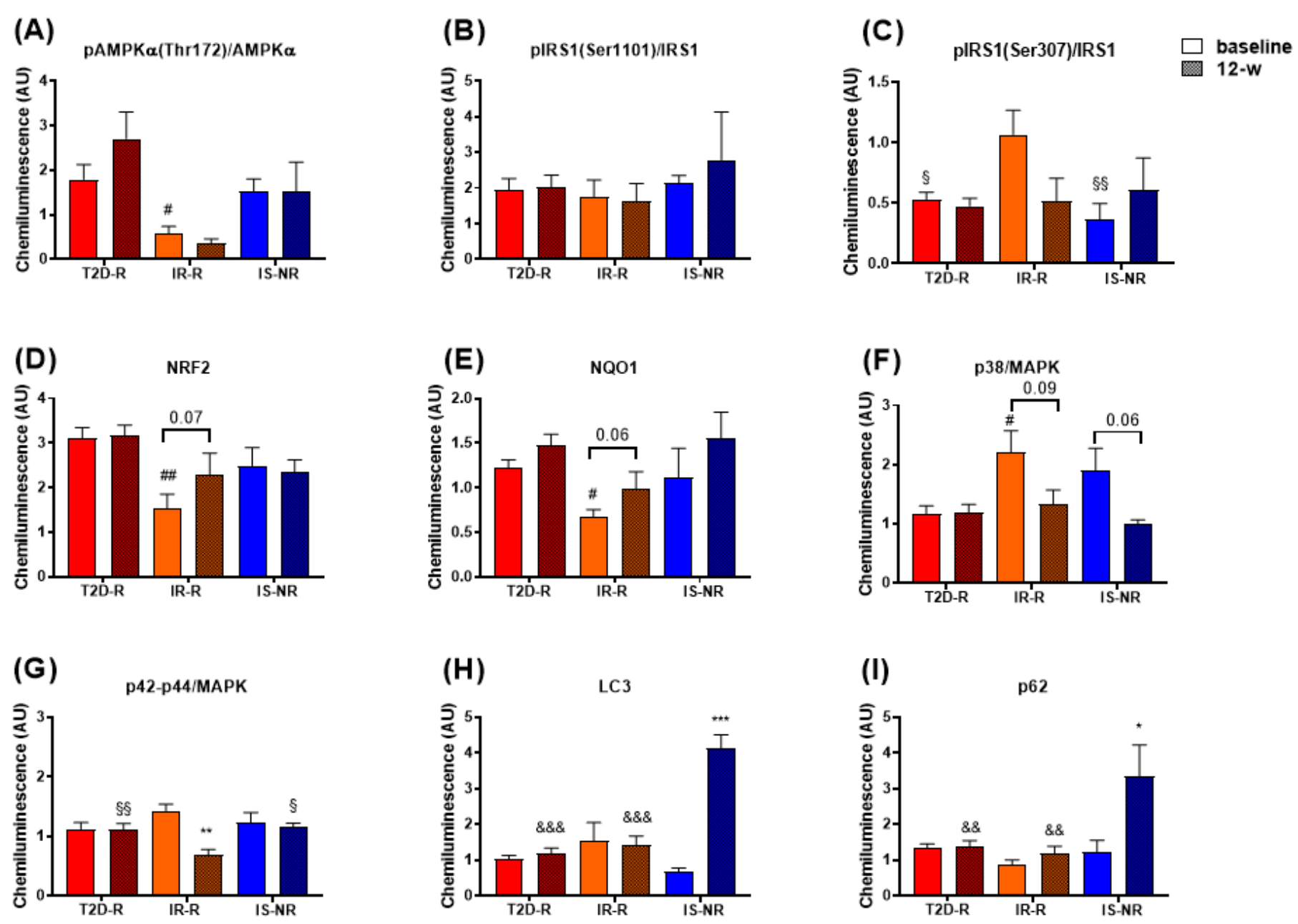

\section{Figure 6}

Selected SEV-cargoed proteins are differentially expressed in skeletal muscle after HIIT. Western blot analysis of pAMPKa(Thr172)/AMPKa (\#p=0.03 T2D-R vs IR-R) (a), pIRS1(Ser1101)/IRS1 (b), pIRS1(Ser307)/IRS1 ( $(p=0.02$ T2D-R vs IR-R, $\S \S p=0.007$ IR-R vs IS-NR) (c), NRF2 (\#\#p=0.001 T2D-R vs IR-R) (d), NQ01 (\#p=0.02 T2D-R vs IR-R) (e), p38/MAPK (\#p=0.01 T2D-R vs IR-R) (f), p42-p44/MAPK $\left(p=0.002\right.$ for IR-R; $\S \S p=0.009$ T2D-R vs IR-R, $\S p=0.02$ IR-R vs IS-NR) (g), LC3 ( ${ }^{\star} \star * p=0.0001$ for IS-NR; $\& \& \& p<0.0001$ IS-NR vs T2D-R and IR-R) (h), p62 ( ${ }^{\star} p=0.02$ for IS-NR; \&\&p=0.003 IS-NR vs T2D-R and IR-R) (i) in muscle biopsies obtained from T2D-R ( $N=15)$, IR-R ( $N=9)$ and IS-NR $(N=7)$ individuals at baseline and after 12-week HIIT. Data are expressed as mean \pm SEM. 
(A)

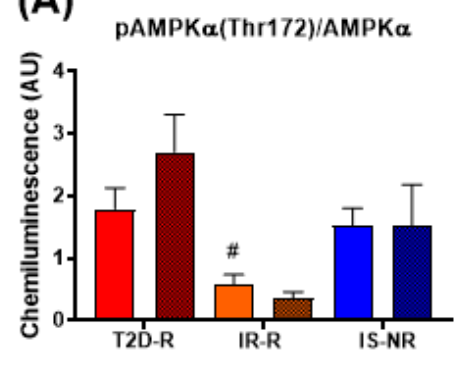

(D)

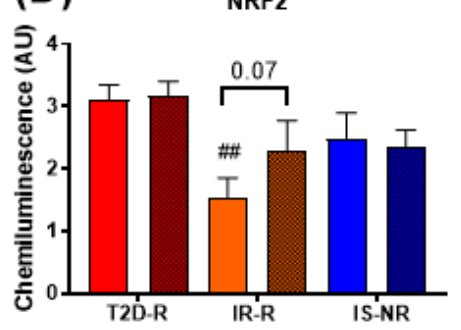

(G)

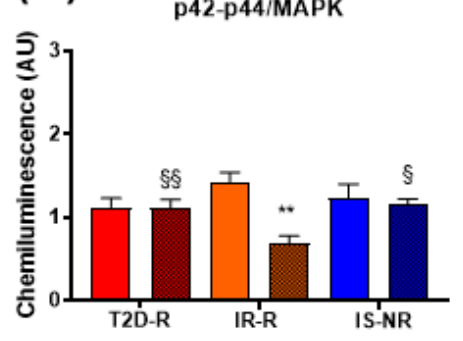

(B)

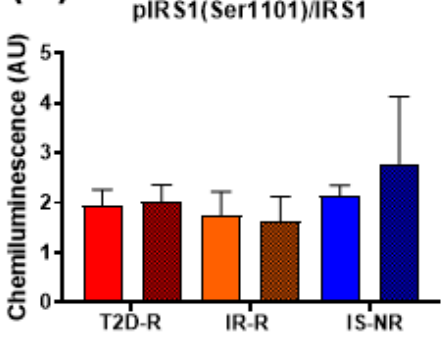

(E)

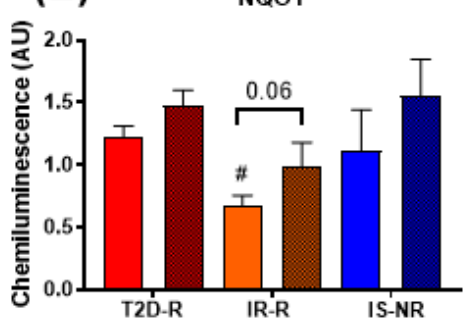

(H)

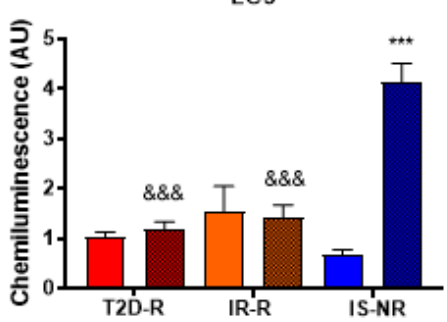

(C)

pIRS1(Ser307)/RRS1

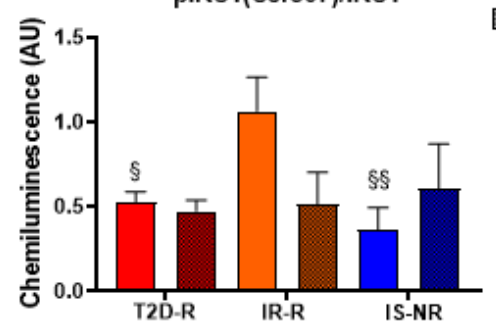

(F) P38/MAPK

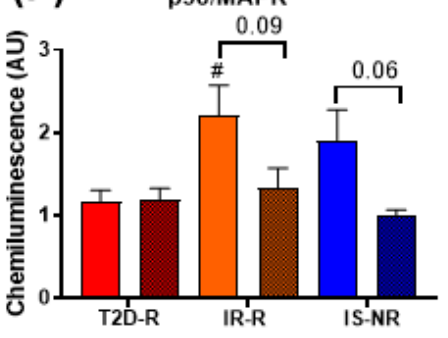

(I)

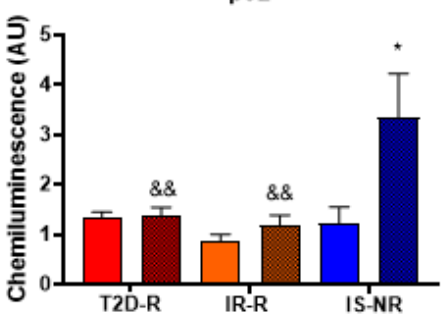

Figure 6

Selected SEV-cargoed proteins are differentially expressed in skeletal muscle after HIIT. Western blot analysis of pAMPKa(Thr172)/AMPKa (\#p=0.03 T2D-R vs IR-R) (a), pIRS1(Ser1101)/IRS1 (b), pIRS1(Ser307)/IRS1 ( $\S p=0.02$ T2D-R vs IR-R, $\S \S p=0.007$ IR-R vs IS-NR) (c), NRF2 (\#\#p=0.001 T2D-R vs IR-R) (d), NQ01 (\#p=0.02 T2D-R vs IR-R) (e), p38/MAPK (\#p=0.01 T2D-R vs IR-R) (f), p42-p44/MAPK $\left(p=0.002\right.$ for IR-R; $\S \S p=0.009$ T2D-R vs IR-R, $\S p=0.02$ IR-R vs IS-NR) (g), LC3 ( ${ }^{\star \star \star} p=0.0001$ for IS-NR; \&\&\&p<0.0001 IS-NR vs T2D-R and IR-R) (h), p62 ( ${ }^{\star} p=0.02$ for IS-NR; \&\&p=0.003 IS-NR vs T2D-R and IR-R) (i) in muscle biopsies obtained from T2D-R ( $N=15)$, IR-R ( $N=9)$ and IS-NR $(N=7)$ individuals at baseline and after 12-week HIIT. Data are expressed as mean \pm SEM.

\section{Supplementary Files}

This is a list of supplementary files associated with this preprint. Click to download.

- supplementalinformationNatCommun.docx

- supplementalinformationNatCommun.docx 
- Tables3Natcommun.xIsx

- Tables3Natcommun.xIsx

- HIITprotocol.docx

- HIITprotocol.docx 